TRANSACTIONS OF THE

AMERICAN MATHEMATICAL SOCIETY

Volume 355, Number 1, Pages 213-239

S 0002-9947(02)03105-7

Article electronically published on September 5, 2002

\title{
UNIQUENESS FOR THE DETERMINATION OF SOUND-SOFT DEFECTS \\ IN AN INHOMOGENEOUS PLANAR MEDIUM BY ACOUSTIC BOUNDARY MEASUREMENTS
}

\author{
LUCA RONDI
}

\begin{abstract}
We consider the inverse problem of determining shape and location of sound-soft defects inside a known planar inhomogeneous and anisotropic medium through acoustic imaging at low frequency. In order to determine the defects, we perform acoustic boundary measurements, with prescribed boundary conditions of different types. We prove that at most two, suitably chosen, measurements allow us to uniquely determine multiple defects under minimal regularity assumptions on the defects and the medium containing them. Finally, we treat applications of these results to the case of inverse scattering.
\end{abstract}

\section{INTRODUCTION}

In this paper we prove some uniqueness results for the following kind of inverse boundary value problem.

Let $\Omega$ be a given bounded planar domain, $A$ a $2 \times 2$ tensor in $\Omega$ satisfying a uniform ellipticity condition and $b$ a measurable positive function in $\Omega$. Let $\Sigma$ be a closed set contained in $\Omega$ such that $\Omega \backslash \Sigma$ is connected.

Then the (direct) boundary value problem consists of finding a function $u$ that solves, for a positive constant $k$,

$$
\begin{cases}\operatorname{div}(A \nabla u)+k b u=0 & \text { in } \Omega \backslash \Sigma, \\ u=0 & \text { on } \Sigma\end{cases}
$$

and satisfies, in addition, a boundary condition on the exterior boundary $\partial \Omega$; namely, either a Dirichlet condition

$$
u=\psi \quad \text { on } \partial \Omega
$$

or a Neumann one

$$
A \nabla u \cdot \nu=\eta \quad \text { on } \partial \Omega .
$$

The inverse boundary value problem of interest to us is the following. Let us assume that the set $\Sigma$ is unknown and our aim is to recover it through additional information on the solutions $u$ of the direct boundary value problems (1.1), (1.2) or (1.1), (1.3). The additional information on $u$ is obtained by performing measurements on a part of the boundary of the region where the medium, in which the unknown defects are buried, is located, that is on $\Gamma$, a subarc of $\partial \Omega$. More precisely,

Received by the editors November 12, 2001 and, in revised form, March 19, 2002.

2000 Mathematics Subject Classification. Primary 35R30.

(C)2002 American Mathematical Society 
we prescribe one or more, suitably chosen, boundary data $\psi$ and we measure on $\Gamma$ the value of $A \nabla u \cdot \nu$, where $u$ is the solution to (1.1), (1.2). Alternatively, we choose and prescribe boundary data $\eta$ and measure the value on $\Gamma$ of the solutions to (1.1), 1.3.

We ask whether these additional measurements determine uniquely the set $\Sigma$; that is, if there is a unique set $\Sigma$ that is compatible with the measurements performed.

The Helmholtz type equation $\operatorname{div}(A \nabla u)+k b u=0$ in $\Omega \backslash \Sigma$ can model the wave pattern of a low amplitude sound wave with a fixed temporal frequency, given by $\sqrt{k}$, in a, possibly inhomogeneous and anisotropic, given medium. The closed set $\Sigma$ is constituted by the finite union of pairwise disjoint closed sets, and the boundary condition $u=0$ on $\Sigma$ can model the presence of a sound-soft multiple defect buried into the medium. Therefore, our inverse problem can be described as an acoustic imaging technique for the detection in a given medium of sound-soft defects or flaws such as, for example, obstacles or cracks.

In fact, there are two cases of particular interest. In the first one, each of the sets constituting $\Sigma$ can be represented as the graph of a simple open curve. Here $\Sigma$ models the presence of many cracks, that is, fractures in the medium. In the second case, the components of $\Sigma$ are regions delimited by simple closed curves, and we model, therefore, the presence of finitely many obstacles in the medium.

There is a well-developed theory, as well as numerical methods, on the determination of defects, such as obstacles or cracks, through inverse scattering techniques associated to acoustic waves; see, for instance, the book by Colton and Kress [8], and the recent survey paper 7] for the obstacle case and 16] for the crack case. Our approach is different since we do not perform measurements in the far field, as in the scattering case, but on the (exterior) boundary of the medium obtaining additional information on the behaviour of the waves on the boundary. However, from the scattering data, it is possible to obtain information on the near field and hence on the boundary data. Therefore, we can apply our results also to the scattering case and we generalize, at least in the planar case, some uniqueness results for the determination of obstacles in a known inhomogeneity contained in [15] by lowering the assumptions on the defects and the inhomogeneity containing them. See Section 4 for details.

Let us describe the main results of the paper, as well as the main assumptions that we make on the data of the problem. We shall keep such assumptions as minimal as possible.

Concerning the unknown multiple defect $\Sigma$, we allow the greatest generality in its definition, in order to cover all the most interesting cases in applications. Namely, we assume only that $\Sigma$ is composed of a finite collection of pairwise disjoint defects, where a defect $\sigma$ in $\Omega$ is a closed continuum contained in $\Omega$ such that $\Omega \backslash \sigma$ is connected. We recall that a continuum is a connected set with at least two points. The previously described cases of obstacles and cracks are clearly included in this broader definition. Indeed, this definition allows a multiple defect to be constituted by both obstacles and cracks at the same time. Furthermore, and more notably, no regularity assumption is made on the components of $\Sigma$, which may not even be curves. Our assumptions, however, guarantee that $\Sigma$ is not reduced to a single point and that $\Sigma$ does not break $\Omega$ into two or more pieces. 
For the medium in which the defects are buried, we do not assume it to be either homogeneous or isotropic; that is, the coefficients of the Helmholtz type equation involved, $A$ and $b$, satisfy only the following. We suppose that $A$ is a measurable, symmetric, bounded and uniformly positive definite $2 \times 2$ matrix in $\Omega$ and $b$ is a measurable, bounded and positive function in $\Omega$. We remark that no continuity or smoothness assumption is imposed on the coefficients.

We shall consider the case of waves at low frequency; that is, we assume that $k$ is positive and less than a constant which depends on known quantities involving the geometry of $\Omega$, the region occupied by the medium, and the physical properties of the medium itself; that is, the coefficients of the Helmholtz type equation, and, in certain cases, on a priori information concerning, roughly speaking, the size of the unknown multiple defect.

Then the following kind of unique determination will be proved, either by prescribing Dirichlet data on $\partial \Omega$ and measuring the corresponding Neumann conditions on an open subarc $\Gamma$ of $\partial \Omega$, or by assigning Neumann conditions on $\partial \Omega$ and measuring the Dirichlet data on $\Gamma$.

First, we show that one measurement is enough to uniquely determine $\Sigma$ in these two cases. If we assume that $\Sigma$ is composed of a collection of obstacles, then the measurement corresponding to any nontrivial prescribed boundary condition is enough for the determination of $\Sigma$; see Theorem 3.2 Otherwise, if we wish to determine a multiple defect $\Sigma$, which might include cracks, with just one measurement, we need to prescribe a nonnegative boundary datum; see Theorem 3.3.

In the third uniqueness result, Theorem 3.4, we treat the case when two measurements are used. In order to uniquely determine the unknown multiple defect $\Sigma$, we can choose two prescribed boundary data so that they satisfy suitable assumptions on their sign changes on $\partial \Omega$, assumptions less restrictive than the one in Theorem 3.3.

The mathematical formulation of our inverse problem is similar to the one of determining a collection of defects, in particular, cracks or cavities, inside a conductor body by performing electrostatic current and voltage measurements on the exterior boundary of the conductor itself. This problem, which has been referred to as the inverse crack problem, was introduced by Friedman and Vogelius who proved the first uniqueness result, 9. Many other authors contributed to establish further uniqueness and stability results; see [20] for references on the subject.

In fact, in order to prove our results, we shall extend to the Helmholtz type equation the technique developed in [2] in order to establish uniqueness results for the inverse crack problem, coupling it sometimes with reasonings close in spirit to some used in [3], where the inverse crack problem in three dimensions has been tackled.

The plan of the paper is as follows. In Section 2 we study qualitative properties of solutions to (1.1). In particular, we introduce a generalized notion of nodal critical point, which follows from the one in 4 for elliptic equations without any lower-order term, and we investigate the relationship between the presence of nodal critical points for $u$, the solution to (1.1), and the behaviour of $u$ on $\partial \Omega$; see Proposition 2.2. In Section 3 we state and prove the uniqueness results for our inverse problems briefly outlined above. The applications to the scattering case are developed in Section 4. 
Acknowledgements. The author wishes to thank Professor Giovanni Alessandrini for useful discussions on the subject of this work. The present work was initiated while the author was at the Institut für Industriemathematik/SFB 013 of the Johannes Kepler Universität Linz, Austria, and was supported by the Austrian Science Foundation FWF under project SFB 013 F1310.

\section{Helmholtz TYPE EQUATION With LOW FREQUENCY IN A MEDIUM CONTAINING SOUND-SOFT DEFECTS}

We study the existence and uniqueness of the solution to the Helmholtz type equation with low frequency in a planar medium, which contains a finite number of sound-soft defects, under different types of boundary conditions on the (exterior) boundary of the medium itself.

Then we shall describe some qualitative properties of these solutions; in particular, we shall investigate the behaviour of their nodal lines, that is, their level lines at level 0; see Proposition 2.2.

For any $z \in \mathbb{R}^{2}$ and any positive number $r$, we denote by $B_{r}(z)$ the open ball with centre $z$ and radius $r$. We also use the following notation for complex derivatives:

$$
f_{\bar{z}}=\frac{1}{2}\left(f_{x}+\mathrm{i} f_{y}\right), \quad f_{z}=\frac{1}{2}\left(f_{x}-\mathrm{i} f_{y}\right),
$$

where $f$ is usually a complex-valued function.

For any planar domain $\Omega$ and any real number $p, p \geq 1, W^{1, p}(\Omega)$ will denote, as usual, the Sobolev space

$$
W^{1, p}(\Omega)=\left\{u \in L^{p}(\Omega): \nabla u \in L^{p}(\Omega)\right\}
$$

where $\nabla u$ denotes the gradient of $u$ in the sense of distributions. By $W_{0}^{1, p}(\Omega)$ we denote the closure in $W^{1, p}(\Omega)$ of $C_{0}^{\infty}(\Omega)$, the space of $C^{\infty}(\Omega)$ functions whose supports are compactly contained in $\Omega$. When $p=2$, we usually denote $W^{1,2}(\Omega)$ by $H^{1}(\Omega)$ and $W_{0}^{1,2}(\Omega)$ by $H_{0}^{1}(\Omega)$. We recall that a domain is said to be Lipschitz if its boundary $\partial \Omega$ can be locally represented as the graph of a Lipschitz function. If $\Omega$ is bounded and Lipschitz, then $H^{1 / 2}(\partial \Omega)$ denotes the trace space of $H^{1}(\Omega)$ on $\partial \Omega$. By $H^{-1 / 2}(\partial \Omega)$ we shall denote the dual space to $H^{1 / 2}(\partial \Omega)$. We recall that $H^{1 / 2}(\partial \Omega) \subset L^{2}(\partial \Omega) \subset H^{-1 / 2}(\partial \Omega)$.

2.1. Capacities. We shall make use of different notions of capacity. We restrict ourselves to the planar case. The first kind of capacity that we introduce is the so-called Sobolev 2-capacity. For any $E \subset \mathbb{R}^{2}$, we set

$$
C_{1,2}(E)=\inf _{u \in S(E)} \int_{\mathbb{R}^{2}}|u|^{2}+|\nabla u|^{2}
$$

where

$$
S(E)=\left\{u \in H^{1}\left(\mathbb{R}^{2}\right): u=1 \text { a.e. in an open set containing } E\right\} .
$$

If $S(E)$ is empty, then $C_{1,2}(E)=\infty$. The number $C_{1,2}(E)$ is called the Sobolev 2-capacity of $E$. For its basic properties we refer, for instance, to [12, Chapter 2]. We recall that if $K$ is a compact set contained in $\mathbb{R}^{2}$, then

$$
C_{1,2}(K)=\inf _{u \in S(K) \cap C_{0}^{\infty}\left(\mathbb{R}^{2}\right)} \int_{\mathbb{R}^{2}}|u|^{2}+|\nabla u|^{2} .
$$

Our interest in capacity follows from the fact that capacity plays an important rôle in the following Poincaré type inequalities. It is well known that, for any 
bounded domain $\Omega \subset \mathbb{R}^{2}$, there exists a constant $C(\Omega)$ such that for any $u \in H_{0}^{1}(\Omega)$, we have

$$
\int_{\Omega} u^{2} \leq C(\Omega) \int_{\Omega}|\nabla u|^{2}
$$

This inequality is called the Poincaré inequality and we have that

$$
C(\Omega) \leq(|\Omega| /(2 \pi))^{1 / 2}
$$

see, for instance, [10, page 164]. Here $|\Omega|$ denotes the Lebesgue measure of $\Omega$. In other words, there exists a constant depending on $|\Omega|$ only such that for any function $u \in H^{1}(\Omega)$ such that $u=0$ in a weak sense on $\partial \Omega$, inequality (2.1) holds. A similar property is satisfied also by the class of $H^{1}(\Omega)$ functions vanishing (in a weak sense) on a given set with positive Sobolev 2-capacity.

Let us state this kind of generalization of Poincaré inequality; see [23. Section 4.5] for details. Let $\Omega$ be a bounded Lipschitz domain and let $\Sigma$ be a closed set contained in $\Omega$ with positive Sobolev 2-capacity. Then there exists a constant $C(\Sigma, \Omega)$ such that

$$
\int_{\Omega} u^{2} \leq C(\Sigma, \Omega) \int_{\Omega}|\nabla u|^{2}
$$

for any $u \in H^{1}(\Omega)$ satisfying $u=0$ in a weak sense on $\Sigma$. Furthermore, we have that

$$
C(\Sigma, \Omega) \leq C\left(C_{1,2}(\Sigma)\right)^{-1 / 2}
$$

where the constant $C$ depends on $\Omega$ only.

We wish to remark that this result, and, in particular, the estimate (2.3), is stated in 23 with $C_{1,2}(\Sigma)$ replaced by $B_{1,2}(\Sigma)$, where for any $E \subset \mathbb{R}^{2}, B_{1,2}(E)$ denotes the (1,2)-Bessel capacity of $E$ (see [23, Section 2.6] for its definition). These two notions of capacity are equivalent; that is, there exist two positive constants $A_{1}$ and $A_{2}$ such that

$$
A_{1} B_{1,2}(E) \leq C_{1,2}(E) \leq A_{2} B_{1,2}(E)
$$

for any set $E \subset \mathbb{R}^{2}$. Therefore, since the definition of Bessel capacity is somewhat involved, we have preferred to use the Sobolev 2-capacity. The equivalency of the two capacities may be found in [1, by coupling Proposition 2.3.13 with the remark after Definition 2.2.6 therein contained.

Formula 2.3 provides an upper bound on the constant $C(\Sigma, \Omega)$ introduced in (2.2) in terms of the Sobolev 2-capacity of $\Sigma$. In order to make our results more applicable, we would like to estimate from above $C(\Sigma, \Omega)$ in terms of metric properties of $\Sigma$, which are easier to compute. For planar sets we shall be able to obtain these estimates using known results concerning another notion of capacity, the logarithmic capacity. For any bounded and closed subset $E$ of $\mathbb{R}^{2}$, let $\gamma(E)$ be its logarithmic capacity. For a definition of logarithmic capacity and its basic properties, we refer to [22, Chapter III]. Our interest in logarithmic capacity relies on the fact that if $\sigma$ is a closed and bounded continuum, then $\gamma(\sigma)>0$; see [22, Theorem III.5]. Furthermore, not only is $\gamma(\sigma)$ positive, but, under the same assumptions on $\sigma$, we also have that

$$
\gamma(\sigma) \geq \frac{\operatorname{diam}(\sigma)}{4}
$$

where $\operatorname{diam}(\sigma)$ denotes the diameter of $\sigma$; see [22, page 85]. 
We shall use (2.4) to estimate $C_{1,2}(\sigma)$ from below where $\sigma$ is a bounded and closed continuum. In order to find a suitable relation between the Sobolev 2-capacity and the logarithmic capacity, we need to consider two other notions of capacity.

The first is defined as follows. For any bounded domain $\Omega \subset \mathbb{R}^{2}$ and any $E \subset \Omega$, we define the 2-capacity of the condenser $(E, \Omega), \operatorname{cap}_{2}(E, \Omega)$, as

$$
\operatorname{cap}_{2}(E, \Omega)=\inf _{u \in S(E, \Omega)} \int_{\Omega}|\nabla u|^{2}
$$

where

$$
S(E, \Omega)=\left\{u \in H_{0}^{1}(\Omega): u=1 \text { a.e. in an open set containing } E\right\} .
$$

If $S(E, \Omega)$ is empty, we set $\operatorname{cap}_{2}(E, \Omega)=\infty$. Also, for this capacity, if $K$ is a compact set contained in $\Omega$, we have

$$
\operatorname{cap}_{2}(K, \Omega)=\inf _{u \in S(E, \Omega) \cap C_{0}^{\infty}(\Omega)} \int_{\Omega}|\nabla u|^{2} .
$$

For basic properties of the 2-capacity of condensers, we refer again to 12, Chapter 2]. Here we simply recall that, for any $x_{0} \in \mathbb{R}^{2}$ and any $0<r<R$, the following formula holds:

$$
\operatorname{cap}_{2}\left(\overline{B_{r}\left(x_{0}\right)}, B_{R}\left(x_{0}\right)\right)=2 \pi(\log (R / r))^{-1},
$$

and that the following relation holds between the Sobolev 2-capacity and the 2capacity of a condenser. There exists a positive constant $A_{3}$ such that for any $E \subset B_{r}\left(x_{0}\right)$, we have

$$
\left(1+A_{3} r^{2}\right)^{-1} C_{1,2}(E) \leq \operatorname{cap}_{2}\left(E, B_{2 r}\left(x_{0}\right)\right) \leq 16\left(1+r^{-2}\right) C_{1,2}(E) ;
$$

see [12 Theorem 2.38].

The other capacity that we need to introduce is the so-called hyperbolic capacity. For any planar closed set $E$ contained in $B_{1}(0)$, we denote with $\gamma_{-}(E)$ the hyperbolic capacity of $E$. For the definition of this capacity we refer to [22] Section III.12]. By the definitions of logarithmic and hyperbolic capacities, it is immediate to remark that for every $r, 0<r<1$, if $E$ is a closed set contained in $B_{r}(0)$, we have

$$
\frac{\gamma(E)}{1+r^{2}} \leq \gamma_{-}(E) \leq \frac{\gamma(E)}{1-r^{2}}
$$

The last link that we need is provided by the modulus of a ring domain; see 22 . Section III.13]. Let $\sigma$ be a closed continuum contained in $B_{1}(0)$. Then we consider the ring domain $B_{1}(0) \backslash \sigma$ and denote by $M\left(B_{1}(0) \backslash \sigma\right)$ its modulus. On one side, we use (2.5) and the fact that capacity of condensers and modulus of ring domains are invariant by conformal mappings to prove that

$$
\operatorname{cap}_{2}\left(\sigma, B_{1}(0)\right)=2 \pi M\left(B_{1}(0) \backslash \sigma\right)^{-1} .
$$

On the other hand, Theorem III.54 in 22] states that

$$
M\left(B_{1}(0) \backslash \sigma\right)=\log 1 / \gamma_{-}(\sigma) .
$$

We collect all of these results as follows. Let $\sigma$ be a closed continuum and let $\operatorname{diam}(\sigma)$ be its diameter. We take $x_{0} \in \sigma$. Without loss of generality, we can assume $x_{0}=0$ and we denote $\tilde{\sigma}=\sigma \cap \overline{B_{1 / 3}(0)}$. It follows that $\tilde{\sigma}$ is a closed continuum 
contained in $B_{1 / 2}(0)$ and, by (2.6) and the monotonicity property of capacity, we infer immediately that

$$
C_{1,2}(\sigma) \geq C_{1,2}(\tilde{\sigma}) \geq \operatorname{cap}_{2}\left(\tilde{\sigma}, B_{1}(0)\right) / 80 .
$$

On the other hand, we have that $\operatorname{diam}(\tilde{\sigma}) \geq \min \{1 / 3, \operatorname{diam}(\sigma)\}$ and hence, by (2.4) and (2.7), we have

$$
\gamma_{-}(\tilde{\sigma}) \geq \frac{4}{5} \gamma(\tilde{\sigma}) \geq \min \left\{\frac{1}{15}, \frac{\operatorname{diam}(\sigma)}{5}\right\} .
$$

By (2.8) and (2.9), we infer that

$$
\operatorname{cap}_{2}\left(\tilde{\sigma}, B_{1}(0)\right)=\frac{2 \pi}{\log 1 / \gamma_{-}(\tilde{\sigma})} .
$$

By collecting the last three equations, we have that for any closed continuum $\sigma$, the following estimate holds:

$$
C_{1,2}(\sigma) \geq \frac{\pi}{-40 \log \left(\min \left\{\frac{1}{15}, \frac{\operatorname{diam}(\sigma)}{5}\right\}\right)} .
$$

Hence we deduce that

$$
C_{1,2}(\sigma) \geq \frac{\pi}{40 \log (\max \{15,5 / \operatorname{diam}(\sigma)\})} .
$$

An immediate corollary of this last result is that a closed continuum $\sigma$ has a strictly positive Sobolev 2-capacity.

2.2. First eigenvalues. Let $\Omega \subset \mathbb{R}^{2}$ be a bounded domain. Let $A=A(z), z \in \Omega$, be a $2 \times 2$ symmetric matrix whose entries are measurable and which satisfies the following uniform ellipticity condition for a positive constant $\lambda$ :

$$
\lambda I d \leq A(z) \leq \lambda^{-1} I d \quad \text { for a.e. } z \in \Omega,
$$

where $I d$ denotes the identity matrix.

Let $b=b(z), z \in \Omega$, be a measurable function satisfying the condition

$$
\lambda \leq b(z) \leq \lambda^{-1} \text { for a.e. } z \in \Omega .
$$

We define the number $k_{0}(\Omega, A, b)$ in the following way:

$$
k_{0}(\Omega, A, b)=\min _{u \in H_{0}^{1}(\Omega), u \neq 0} \frac{\int_{\Omega} A \nabla u \cdot \nabla u}{\int_{\Omega} b u^{2}} .
$$

We have that $k_{0}$ is a positive number, and it is the first Dirichlet eigenvalue associated to the following boundary value problem:

$$
\begin{cases}-\operatorname{div}(A \nabla u)=k b u & \text { in } \Omega, \\ u=0 & \text { on } \partial \Omega .\end{cases}
$$

We remark that the following monotonicity property holds:

$$
k_{0}(\Omega, A, b) \leq k_{0}\left(\Omega_{1}, A, b\right) \text { for any domain } \Omega_{1} \subset \Omega .
$$

We also notice that if $A \equiv I d$ and $b \equiv 1$, we have that $k_{0}(\Omega, I d, 1)$ is the first eigenvalue of the Laplace operator and the following inequality holds:

$$
\lambda^{2} k_{0}(\Omega, I d, 1) \leq k_{0}(\Omega, A, b) \leq \lambda^{-2} k_{0}(\Omega, I d, 1) .
$$


Finally, it is evident that $k_{0}(\Omega, I d, 1)$ is given by $1 / C(\Omega)$ where $C(\Omega)$ is the best possible constant in (2.1). Therefore, $k_{0}(\Omega, A, b)$ satisfies the estimate

$$
k_{0}(\Omega, A, b) \geq \frac{\lambda^{2}}{C(\Omega)} \geq \lambda^{2}\left(\frac{2 \pi}{|\Omega|}\right)^{1 / 2} .
$$

For a review of theoretical results, as well as numerical methods, concerning the Dirichlet eigenvalues of the Laplace operator for 2-dimensional domains, we refer to [17.

For any closed set $\Sigma$ contained in $\Omega$, we define the number $k_{0}(\Sigma, \Omega, A, b)$ as

$$
k_{0}(\Sigma, \Omega, A, b)=\min _{u \in H^{1}(\Sigma, \Omega), u \neq 0} \frac{\int_{\Omega} A \nabla u \cdot \nabla u}{\int_{\Omega} b u^{2}}
$$

where $H^{1}(\Sigma, \Omega)$ denotes the set of $H^{1}(\Omega)$ functions vanishing (in a weak sense) on $\Sigma$. We observe that if the Sobolev 2-capacity of $\Sigma$ is zero, then $H^{1}(\Sigma, \Omega)$ coincides with $H^{1}(\Omega)$ and therefore $k_{0}(\Sigma, \Omega, A, b)=0$ in this case. On the other hand, if $C_{1,2}(\Sigma)>$ 0 , we have that $k_{0}(\Sigma, \Omega, A, b)$ is strictly positive and is linked to $k_{0}(\Sigma, \Omega, I d, 1)$ by a formula completely analogous to (2.16). Furthermore, it is possible to evaluate $k_{0}(\Sigma, \Omega, I d, 1)$ in terms of the best constant $C(\Sigma, \Omega)$ appearing in the Poincaré type inequality (2.2). As before, $k_{0}(\Sigma, \Omega, I d, 1)=1 / C(\Sigma, \Omega)$. Therefore, using (2.3),$k_{0}(\Sigma, \Omega, A, b)$ can be bounded from below by a positive constant depending on $\lambda, \Omega$ and the Sobolev 2-capacity of $\Sigma$ only, as follows:

$$
k_{0}(\Sigma, \Omega, A, b) \geq \frac{\lambda^{2}}{C(\Sigma, \Omega)} \geq \lambda^{2} C\left(C_{1,2}(\Sigma)\right)^{1 / 2}
$$

where $C$ is a positive constant depending on $\Omega$ only.

2.3. Nodal critical points. Given a bounded domain $\Omega$, we say that $\sigma \subset \Omega$ is a defect in $\Omega$ if $\sigma$ is a closed continuum such that $\Omega \backslash \sigma$ is connected.

A multiple defect in $\Omega$ will be a subset $\Sigma$ of $\Omega$ that is the union of a finite number of pairwise disjoint defects, $\sigma_{1}, \ldots, \sigma_{N}$, where $N$ is a positive integer. We have that $\Omega \backslash \Sigma$ is a connected open set and we have already noticed that $\Sigma$ has a strictly positive Sobolev 2-capacity. We introduce the following notation. Given a multiple defect $\Sigma=\bigcup_{i=1}^{N} \sigma_{i}$, we set

$$
\delta(\Sigma)=\max _{i=1, \ldots, N}\left\{\operatorname{diam}\left(\sigma_{i}\right)\right\} .
$$

In order to be able to compare the case when no defect is present in the medium, with a slight abuse of notation, we consider also the empty set as a multiple defect. We assume that

$$
\delta(\Sigma)=0 \quad \text { if } \Sigma=\emptyset .
$$

Therefore, in the sequel of the paper, we shall always assume that $\Sigma$ is either the empty set or a finite union of (nonempty) pairwise disjoint defects contained in $\Omega$.

We are interested in functions $u$ satisfying in a weak sense, for a given positive constant $k$, the following:

$$
\begin{cases}\operatorname{div}(A \nabla u)+k b u=0 & \text { in } \Omega \backslash \Sigma, \\ u=0 & \text { on } \Sigma ;\end{cases}
$$

that is, functions $u$ belonging to $H^{1}(\Sigma, \Omega)$ such that

$$
\int_{\Omega \backslash \Sigma} A \nabla u \cdot \nabla \phi-k b u \phi=0 \quad \text { for any } \phi \in H_{0}^{1}(\Omega \backslash \Sigma) \text {. }
$$


First, we notice that functions $u$ satisfying (2.20) are continuous functions in $\Omega$. If $\Sigma$ is empty, this may be deduced by standard regularity estimates in the interior for elliptic equations. If $\Sigma$ is not empty, every component of $\Sigma$ being a continuum, we deduce that every point of $\partial \Sigma$ is regular for the Dirichlet problem for the Laplace equation with respect to the domain $\Omega \backslash \Sigma$; see, for instance, [22]. Hence, by standard regularity estimates in the interior and [18], we obtain that any solution $u$ to $(2.20)$ belongs to $C(\Omega)$ and hence satisfies $u=0$ on $\Sigma$ also in a classical sense.

Weak solutions to an equation of the kind $\operatorname{div}(A \nabla u)+k b u=0$, with $k$ a positive constant, can be related, at least locally, to weak solutions to an elliptic equation in divergence form with no lower-order terms, that is, to functions $\tilde{u}$ satisfying $\operatorname{div}(\tilde{A} \nabla \tilde{u})=0$, with $\tilde{A}$ a symmetric matrix satisfying the uniform ellipticity condition (2.11).

Given a simply connected, bounded, Lipschitz domain $D$, a symmetric and measurable matrix $A$ satisfying (2.11) in $D$, a measurable function $b$ satisfying (2.12) in $D$, we assume that $u \in H^{1}(D) \cap C(\bar{D})$ solves, in a weak sense,

$$
\operatorname{div}(A \nabla u)+k b u=0 \text { in } D,
$$

where $k$ is a positive constant so that $k<k_{0}(D, A, b)$. We remark that $k<$ $k_{0}(D, A, b)$ provided that the measure of $D$ is small enough. Hence all the following considerations might be applied locally inside $\Omega \backslash \Sigma$ for solutions to (2.20). For such a $k$, we have that there exists a unique weak solution $w$ to the boundary value problem

$$
\begin{cases}\operatorname{div}(A \nabla w)+k b w=0 & \text { in } D, \\ w=1 & \text { on } \partial D .\end{cases}
$$

We have that $w$ is continuous up to the boundary of $D$ and, by the strong maximum principle, is strictly positive in $D$. We consider the function $\tilde{u}=u / w$. We have that $\tilde{u} \in H^{1}(D) \cap C(\bar{D})$ and solves the equation

$$
\operatorname{div}(\tilde{A} \nabla \tilde{u})=0 \text { in } D,
$$

where $\tilde{A}=w^{2} A$. We notice that $\tilde{A}$ is measurable, symmetric and satisfies a uniform ellipticity condition in $D$; that is, it satisfies 2.11 for a positive $\lambda$.

We also introduce the function $\tilde{v}$, the so-called stream function associated to $\tilde{u}$, namely, a function satisfying almost everywhere in $D$,

$$
\nabla \tilde{v}=\left[\begin{array}{cc}
0 & -1 \\
1 & 0
\end{array}\right] \tilde{A} \nabla \tilde{u} .
$$

The existence of a single-valued function satisfying this condition is guaranteed by the fact that $D$ is simply connected. The stream function is unique up to an additive constant and, furthermore, we have that $\tilde{v}$ is still a solution to an elliptic equation; namely, $\tilde{v}$ solves

$$
\operatorname{div}(\tilde{B} \nabla \tilde{v})=0 \text { in } D,
$$

where $\tilde{B}=(\operatorname{det} \tilde{A})^{-1} \tilde{A}$. Clearly, also $\tilde{B}$ satisfies the uniform ellipticity condition (2.11). We remark that if $\tilde{u}$ is harmonic, then the notion of stream function coincides with the one of harmonic conjugate and, in this case, the complex-valued function $\tilde{f}=\tilde{u}+\mathrm{i} \tilde{v}$ is known to be holomorphic, that is, to satisfy $\tilde{f}_{\bar{z}}=0$. In the more general case, we have that $\tilde{f}$ satisfies a first-order Beltrami type equation of the following kind:

$$
\tilde{f}_{\bar{z}}=\mu \tilde{f}_{z}+\nu \overline{\tilde{f}_{z}}
$$


where $\mu$ and $\nu$ are measurable complex-valued coefficients in $D$ satisfying almost everywhere in $D$,

$$
|\mu|+|\nu| \leq \kappa<1
$$

where $\kappa$ is a constant strictly less than one depending on the ellipticity and boundedness constants of $\tilde{A}$ only.

Any $H^{1}(D, \mathbb{C})$ solution $\tilde{f}$ of an equation of the type (2.25), (2.26),$\kappa$ being a constant such that $0 \leq \kappa<1$, is called a $\kappa$-quasiconformal function. If, in addition, $\tilde{f}$ is univalent, then $\tilde{f}$ is called a $\kappa$-quasiconformal mapping. We call any $\kappa$-quasiconformal function (respectively mapping) a quasiconformal function (respectively mapping), for some $\kappa, 0 \leq \kappa<1$.

For solutions to an elliptic equation in two dimensions like (2.24), we can define, following [4], a generalized notion of a critical point. Let $\tilde{u}$ solve (2.24) and let $\tilde{v}$ be its associated stream function. A representation theorem due to Bers and Nirenberg (see [6] and also [5]) states that the quasiconformal function $\tilde{f}=\tilde{u}+\mathrm{i} \tilde{v}$ can be represented as $\tilde{f}=\tilde{F} \circ \chi$ where $\chi$ is a quasiconformal mapping, such that $\chi$ and its inverse $\chi^{-1}$ are Hölder continuous, and $\tilde{F}$ is a holomorphic function. If $\tilde{f}=\tilde{u}+\mathrm{i} \tilde{v}$ is not constant, then $z_{0} \in D$ is called a geometrical critical point for $\tilde{u}$, and at the same time for $\tilde{v}$, if $\chi\left(z_{0}\right)$ is a critical point in the classical sense for $\tilde{U}=\Re \tilde{F}$. We remark that the definition is independent of the choice of the representation and coincides with the classical one if $\tilde{u}$ is smooth.

To any geometrical critical point we can also associate a generalized notion of index. Let $G$ be a smooth planar domain and let $E$ be a smooth vector field such that $E \neq 0$ on $\partial G$. Then we define the index of $E$ in $G, I(G, E)$, as - (winding number) of $E$ along $\partial G$; that is,

$$
I(G, E)=-\frac{1}{2 \pi} \int_{\partial G} \mathrm{~d} \arg (E) .
$$

If $z_{0}$ is an isolated zero of $E$, the index of $E$ at $z_{0}$ is given by

$$
I\left(z_{0}, E\right)=\lim _{r \rightarrow 0} I\left(B_{r}\left(z_{0}\right), E\right) .
$$

For the present purposes, a complex-valued function $g=g_{1}+\mathrm{i} g_{2}$ will be identified with the vector field $E=\left(\begin{array}{l}g_{1} \\ g_{2}\end{array}\right)$.

The geometric index of $\nabla \tilde{u}$ at $z_{0} \in D$, still denoted by $I\left(z_{0}, \nabla \tilde{u}\right)$, will be defined as the index of $\nabla \tilde{U}$ at $\chi\left(z_{0}\right)$. We remark that, by this definition, for solutions to elliptic equations like (2.24) the index is positive if and only if $z_{0}$ is a geometrical critical point. Moreover, we have that if $z_{0}$ is such that $\tilde{f}\left(z_{0}\right)=0$, then

$$
I\left(z_{0}, \tilde{f}\right)=I\left(\chi\left(z_{0}\right), \tilde{F}\right)=I\left(\chi\left(z_{0}\right), \nabla \tilde{U}\right)+1=I\left(z_{0}, \nabla \tilde{u}\right)+1 .
$$

Let us also recall that the number of critical points inside a given domain, when they are counted with their multiplicities, is continuous with respect to $H^{1}$ convergence; see 4 for details.

We may give a geometric characterization of the geometric index in the following way. The geometric index of $\nabla \tilde{u}$ at $z_{0}$ is $n, n \geq 0$, if and only if, locally in a neighbourhood of $z_{0}$, the level set $\left\{\tilde{u}=\tilde{u}\left(z_{0}\right)\right\}$ is constituted by $n+1$ simple curves intersecting at $z_{0}$ only. Then, since $u$ and $\tilde{u}$ share the behaviour of the nodal lines, we can define nodal geometrical critical points for solutions to (2.22), along with their indexes, by using the geometrical characterization. That is, $z_{0} \in D$ is a nodal geometrical critical point of index $n, n \geq 1$, for a solution $u$ to (2.22) if the set 
$\left\{u=u\left(z_{0}\right)=0\right\}$ is constituted, in a suitable neighbourhood of $z_{0}$, by $n+1$ simple curves intersecting at $z_{0}$ only. Moreover, the following proposition describing the local behaviour of the nodal lines can be proved.

Proposition 2.1. Let $u$ be a solution of (2.22) where $D$ is a bounded domain, $k$ is a positive number and $A$ and $b$ satisfy the previously stated assumptions, in particular, they verify (2.11) and (2.12) respectively. Let $z_{0}$ be a point of $D$ such that $u\left(z_{0}\right)=0$.

Then there exist a nonnegative integer, $n$, and an open neighbourhood $U$ of $z_{0}$, such that the following holds.

The set $\{z \in U: u(z)=0\}$ is composed of $n+1$ simple curves intersecting at $z_{0}$ only, and the set $\{z \in U: u(z) \neq 0\}$ has exactly $2(n+1)$ connected components $D_{1}, \ldots, D_{2(n+1)}$. Moreover, we can find a Jordan curve $\gamma$ surrounding $z_{0}$ and contained in $U$ such that $\gamma \cap D_{j}$ is connected for every $j$. By following the parametrization on $\gamma$, we can assume that the open sets $D_{j}, j=1, \ldots, 2(n+1)$, are ordered in a clockwise sense. We have that for any $j, j=1, \ldots, 2(n+1)$, the sign of $u$ on $D_{j}$ is different from the sign of $u$ on $D_{j+1}$. Obviously, we identify $D_{2(n+1)+1}$ with $D_{1}$.

Proof. We can fix $r>0$ small enough so that $\overline{B_{r}\left(z_{0}\right)} \subset D$ and $k<k_{0}\left(B_{r}\left(z_{0}\right), A, b\right)$. Let $w$ be the solution to (2.23) when $D$ is replaced by $B_{r}\left(z_{0}\right)$. The function $\tilde{u}=$ $u / w$ satisfies in $B_{r}\left(z_{0}\right)$ the elliptic equation (2.24). Let $\tilde{v}$ be the stream function associated with $\tilde{u}$ in $B_{r}\left(z_{0}\right)$. We have that $\tilde{u}\left(z_{0}\right)=0$ and, without loss of generality, we can assume $\tilde{v}\left(z_{0}\right)=0$. We know that $\tilde{f}=\tilde{u}+\mathrm{i} \tilde{v}$, up to a quasiconformal change of coordinates, is holomorphic. Then, setting $n$ as the index of $\nabla \tilde{u}$ at $z_{0}$, the proposition follows from well-known properties of harmonic and holomorphic functions.

Again, let $D$ be a Lipschitz bounded domain and let $u$ and $u^{\prime}$ belong to $H^{1}(D)$. Let $\Gamma$ be an open subarc of $\partial D$. Then we say that $u=u^{\prime}$ in a weak sense on $\Gamma$ if for any $\phi \in C_{0}^{\infty}\left(\mathbb{R}^{2}\right)$ such that $\operatorname{supp}(\phi) \cap \partial D \subset \Gamma$ we have that $\left(u-u^{\prime}\right) \phi \in H_{0}^{1}(D)$. So $u=0$ on $\Gamma$ if $u \phi \in H_{0}^{1}(D)$ for any $\phi \in C_{0}^{\infty}\left(\mathbb{R}^{2}\right)$ such that $\operatorname{supp}(\phi) \cap \partial D \subset \Gamma$.

For any solution $u$ to (2.22), where in this case we allow $k$ to be either zero or positive, we can define the conormal derivative of $u$ on the boundary, $A \nabla u \cdot \nu, \nu$ denoting the outer normal to $\partial D$, as an element of $H^{-1 / 2}(\partial D)$ such that $(A \nabla u$. $\nu)\left[\left.\phi\right|_{\partial D}\right]=\int_{D} A \nabla u \cdot \nabla \phi-k b u \phi$ for any $\phi \in H^{1}(D)$.

If $u$ and $u^{\prime}$ satisfy (2.22) with the same $k, k \geq 0$, we say that $A \nabla u \cdot \nu=A \nabla u^{\prime} \cdot \nu$ in a weak sense on an open subarc $\Gamma$ of $\partial D$ if $\left(A \nabla u \cdot \nu-A \nabla u^{\prime} \cdot \nu\right)\left[\left.\phi\right|_{\partial D}\right]=0$ for any $\phi \in C_{0}^{\infty}\left(\mathbb{R}^{2}\right)$ such that $\operatorname{supp}(\phi) \cap \partial D \subset \Gamma$. Hence we say that $A \nabla u \cdot \nu=$ 0 in a weak sense on $\Gamma$ if $(A \nabla u \cdot \nu)\left[\left.\phi\right|_{\partial D}\right]=0$ for any $\phi \in C_{0}^{\infty}\left(\mathbb{R}^{2}\right)$ such that $\operatorname{supp}(\phi) \cap \partial D \subset \Gamma$. We say also that $A \nabla u \cdot \nu \geq 0$ ( $\leq 0$ respectively) in a weak sense on $\Gamma$ if $(A \nabla u \cdot \nu)\left[\left.\phi\right|_{\partial D}\right] \geq 0$ ( $\leq 0$ respectively) for any $\phi \in C_{0}^{\infty}\left(\mathbb{R}^{2}\right)$ such that $\operatorname{supp}(\phi) \cap \partial D \subset \Gamma$ and $\phi \geq 0$.

It has been proven in [4] that if two solutions of (2.22) with $k=0$ are such that $u=u^{\prime}$ and $A \nabla u \cdot \nu=A \nabla u^{\prime} \cdot \nu$ on an open subarc $\Gamma$ in a weak sense, then $u=u^{\prime}$ in $D$. This result can be extended to the case when $k>0$ simply by the following considerations.

We can extend $A$ and $b$ on the whole plane by taking $A \equiv I d$ outside $D$ and $b \equiv 1$ outside $D$. We consider a point $z$ in $\Gamma$ and we consider the ball $B_{r}(z)$. We take $r$ small enough in such a way that $k<k_{0}\left(B_{r}(z), A, b\right)$. Hence the problem (2.23), 
with $D$ replaced by $B_{r}(z)$, admits a unique positive solution $w$. It is well known, since [19], that this solution is continuous inside $B_{r}(z)$ and belongs to $W_{\text {loc }}^{1, p}\left(B_{r}(z)\right)$ for some $p>2$. Then, if we take $r_{1}<r$, we have that $\tilde{u}=u / w$ and $\tilde{u}^{\prime}=u^{\prime} / w$ belong to $H^{1}\left(D \cap B_{r_{1}}(z)\right)$. It is also clear that if $u=u^{\prime}$ on $\Gamma$, then $\tilde{u}=\tilde{u}^{\prime}$ on $\Gamma \cap B_{r_{1}}(z)$ in a weak sense.

Furthermore, it is straightforward to notice that if $u=u^{\prime}$ and $A \nabla u \cdot \nu=A \nabla u^{\prime} \cdot \nu$ on $\Gamma$, then not only does $\tilde{u}=\tilde{u}^{\prime}$ on $\Gamma \cap B_{r_{1}}(z)$, but also $\tilde{A} \nabla \tilde{u} \cdot \nu=\tilde{A} \nabla \tilde{u}^{\prime} \cdot \nu$ on $\Gamma \cap B_{r_{1}}(z)$. Therefore, $\tilde{u}=\tilde{u}^{\prime}$ in $B_{r_{1}}(z) \cap D$ and so $u=u^{\prime}$ on this set. So far, we have obtained a local unique continuation property near the boundary. However, we can immediately deduce a global unique continuation property, that is, we can infer that $u=u^{\prime}$ on the whole domain $D$. In fact, it is well known that any solution to (2.22) vanishing in an open subset of $D$ must vanish everywhere; hence, since $u=u^{\prime}$ in an open subset of $D$, they must coincide everywhere.

We have obtained some information concerning the local behaviour of the nodal lines of solutions to (2.20). For our purposes we need to study also a global behaviour of the nodal lines of these functions. In particular, we want to extract information about the behaviour of these solutions on the exterior boundary $\partial \Omega$ from information about their nodal critical points inside $\Omega \backslash \Sigma$.

We shall investigate two kinds of boundary conditions, namely, Dirichlet and Neumann. We assume that $\Omega$ is a bounded, simply connected domain and we assume $\partial \Omega$ to be Lipschitz. Let $\Sigma$ be a multiple defect in $\Omega$. We recall that $\Sigma$ might be the empty set.

Let $\psi \in H^{1}(\Omega)$ and let $k$ be such that $0<k<k_{0}(\Omega \backslash \Sigma, A, b)$. Then the following Dirichlet type boundary value problem admits a unique weak solution:

$$
\begin{cases}\operatorname{div}(A \nabla u)+k b u=0 & \text { in } \Omega \backslash \Sigma \\ u=0 & \text { on } \Sigma \\ u=\psi & \text { on } \partial \Omega\end{cases}
$$

The weak formulation of (2.27) is the following. We look for a function $u \in$ $H^{1}(\Sigma, \Omega)$ such that its trace on $\partial \Omega$ is equal to the trace of $\psi$ on $\partial \Omega$ and such that

$$
\int_{\Omega \backslash \Sigma} A \nabla u \cdot \nabla \phi-k b u \phi=0 \quad \text { for any } \phi \in H_{0}^{1}(\Omega \backslash \Sigma) .
$$

If, in addition, we have that $\psi \in H^{1}(\Omega) \cap C(\bar{\Omega})$, then we infer that $u \in C(\bar{\Omega})$ (see for instance [10, Theorem 8.31]) and $u=\psi$ on $\partial \Omega$ is satisfied in a classical sense.

We consider also the case when a Neumann type condition is imposed on $\partial \Omega$. We assume that $\Sigma$ is not empty and we take $k$ such that $0<k<k_{0}(\Sigma, \Omega, A, b)$. For any $\eta \in H^{-1 / 2}(\partial \Omega)$, we have that the mixed type boundary value problem

$$
\begin{cases}\operatorname{div}(A \nabla v)+k b v=0 & \text { in } \Omega \backslash \Sigma, \\ v=0 & \text { on } \Sigma \\ A \nabla v \cdot \nu=\eta & \text { on } \partial \Omega\end{cases}
$$

admits a unique (weak) solution. The weak formulation of (2.28) is to find a function $v \in H^{1}(\Sigma, \Omega)$ such that

$$
\int_{\Omega \backslash \Sigma} A \nabla v \cdot \nabla \phi-k b v \phi=\eta\left[\left.\phi\right|_{\partial \Omega}\right] \quad \text { for any } \phi \in H^{1}(\Sigma, \Omega) \text {. }
$$


We recall that if $\eta \in L^{2}(\partial \Omega)$, then $\eta \in H^{-1 / 2}(\partial \Omega)$ and we have $\eta[\phi]=\int_{\partial \Omega} \eta \phi$ for any $\phi \in H^{1 / 2}(\partial \Omega)$. Also, if $\eta \in L^{2}(\partial \Omega)$, then $v$ is continuous up to the boundary of $\Omega$; that is, $v \in C(\bar{\Omega})$.

The proof of this continuity result can be sketched as follows. We already know that $v$ is continuous in all $\Omega$. We need only to consider its continuity near $\partial \Omega$. Let us take a smooth simply connected domain $\Omega_{1}$ that is compactly contained in $\Omega$ and such that $\Sigma \subset \Omega_{1}$. Then the Neumann problem

$$
\begin{cases}\operatorname{div}(A \nabla \hat{v})=0 & \text { in } \Omega \backslash \overline{\Omega_{1}}, \\ A \nabla \hat{v} \cdot \nu=\eta & \text { on } \partial \Omega, \\ A \nabla \hat{v} \cdot \nu=-\frac{1}{\left|\partial \Omega_{1}\right|} \int_{\partial \Omega} \eta & \text { on } \partial \Omega_{1}\end{cases}
$$

admits a solution that is unique up to additive constants. Here $\left|\partial \Omega_{1}\right|$ denotes the length of $\partial \Omega_{1}$ and the integral $\int_{\partial \Omega} \eta$ is taken with respect to arclength. We have that $\hat{v}$ is continuous on $\bar{\Omega} \backslash \Omega_{1}$. This may be proved as follows. We study the behaviour of $\hat{v}$ locally near a point of the boundary of $\Omega \backslash \overline{\Omega_{1}}$, which is given by $\partial \Omega \cup \partial \Omega_{1}$. Let us fix a point $z_{0}$ in $\partial \Omega$ and let us investigate the continuity of $\hat{v}$ in a neighbourhood of $z_{0}$. The same kind of reasoning can be applied to points belonging to $\partial \Omega_{1}$. There is no guarantee that a global single-valued stream function associated to $\hat{v}$ exists in $\Omega \backslash \overline{\Omega_{1}}$, since this domain is doubly connected. However, for a fixed $z_{0} \in \partial \Omega$, we can find a neighbourhood $U$ of $z_{0}$ such that $U \cap\left(\Omega \backslash \overline{\Omega_{1}}\right)$ is a simply connected Lipschitz domain. Therefore, $\hat{w}$, the stream function associated to $\hat{v}$ in $U \cap\left(\Omega \backslash \overline{\Omega_{1}}\right)$, is well-defined and single-valued and its trace on $\partial \Omega \cap U$ is equal to $\psi$, the antiderivative of $\eta$ along $\partial \Omega$; that is, $\psi=\int \eta$ where the indefinite integral is taken with respect to arclength in the counterclockwise direction. We remark that $\psi$ is not necessarily single-valued on the whole $\partial \Omega$ (unless, actually, $\eta$ has zero mean) but it is well-defined on every proper open subset of it, and, furthermore, on any such set is Hölder continuous. Then by standard regularity estimates up to the boundary, we infer that $\hat{w}$ is Hölder continuous on $\overline{U_{1} \cap \Omega}$, where we assume $U_{1}$ to be an open neighbourhood of $z_{0}$ compactly contained in $U$ and such that $U_{1} \cap \Omega$ is a simply connected Lipschitz domain. Through a bi-Lipschitz transformation between $U_{1} \cap \Omega$ and $B_{1}(0)$ and the representation theorem already cited, [6], we have that $\hat{v}+\mathrm{i} \hat{w}=\hat{F} \circ \chi$ where $\hat{F}$ is holomorphic in $B_{1}(0)$ and $\chi$ is a quasiconformal mapping between $B_{1}(0)$ and $U_{1} \cap \Omega$ such that $\chi$ and its inverse are Hölder continuous up to the boundary. We deduce that $\hat{W}=\Im \hat{F}$ is Hölder continuous up to $\partial B_{1}(0)$, since $\hat{w}$ is Hölder continuous up to $\partial\left(U_{1} \cap \Omega\right)$. Therefore, by Privaloff's Theorem (see [5, Part II, Chapter 6, Theorem 5, p. 279]), $\hat{V}=\Re \hat{F}$ is also Hölder continuous up to the boundary of $B_{1}(0)$, and this, in turn, implies that $\hat{v}$ is Hölder continuous up to the boundary of $U_{1} \cap \Omega$.

Let us take $\Omega_{2}$ to be a smooth simply connected domain containing the closure of $\Omega_{1}$ and contained (compactly) in $\Omega$. Then by the already-mentioned theorem by Meyers, [19], and by standard regularity estimates in the interior, we can find a function $h$ such that $h$ is continuous in $\mathbb{R}^{2}$ with compact support contained in $\Omega \backslash \overline{\Omega_{1}}, h \in W^{1, q}(\Omega)$ for some $q>2$ and $h=v-\hat{v}$ on $\partial \Omega_{2}$.

Then we have that $h_{1}=v-\hat{v}-h$ satisfies the boundary value problem

$$
\begin{cases}\operatorname{div}\left(A \nabla h_{1}\right)=g+\operatorname{div}(G) & \text { in } \Omega \backslash \overline{\Omega_{2}}, \\ A \nabla h_{1} \cdot \nu=0 & \text { on } \partial \Omega, \\ h_{1}=0 & \text { on } \partial \Omega_{2},\end{cases}
$$


where $g \in L^{p}\left(\Omega \backslash \overline{\Omega_{2}}\right)$ for any $p<\infty$ and $G$ belongs to $L^{q}\left(\Omega \backslash \overline{\Omega_{2}}\right)$ for some $q>2$. A bi-Lipschitz change of variables transforming $\Omega \backslash \overline{\Omega_{2}}$ onto an annulus does not modify the nature of the elliptic equation, of its right-hand side and of its boundary conditions. So, we can assume, without loss of generality, that the boundary of $\Omega \backslash \overline{\Omega_{2}}$ is at least $C^{1}$ and we can apply a theorem by Stampacchia, [21], to ensure that $h_{1} \in C\left(\bar{\Omega} \backslash \Omega_{2}\right)$. Since $v=h_{1}+\hat{v}$ near $\partial \Omega$ and both $h_{1}$ and $\hat{v}$ are continuous up to $\partial \Omega$, the result is proven.

Let $u$ be a solution to (2.20), with $k$ small enough. Then the geometric index of a zero of $u$ provides information on the behaviour of $u$ on the boundary. Roughly speaking, we can estimate the number of sign changes either of $\left.u\right|_{\partial \Omega}$ or of the conormal derivative of $u$ on $\partial \Omega$. We state this result in the following proposition.

Proposition 2.2. Let $u$ be a nonconstant $C(\bar{\Omega}) \cap H^{1}(\Omega)$ solution to (2.20). Let $z_{0} \in \Omega \backslash \Sigma$ be such that $u\left(z_{0}\right)=0$ and let $n$ be the geometric index of $\nabla u$ at $z_{0}$.

Then the following holds:

i) if $k<k_{0}(\Omega \backslash \Sigma, A, b)$, there exist $2(n+1)$ points on $\partial \Omega, y_{j}, j=1, \ldots, 2(n+$ $1)$, ordered in a clockwise sense along $\partial \Omega$, such that $(-1)^{j} u\left(y_{j}\right)>0$ for any $j, j=1, \ldots, 2(n+1)$;

ii) if $k<k_{0}(\Sigma, \Omega, A, b)$, then there exist $2(n+1)$ open, connected and pairwise disjoint subarcs of $\partial \Omega, \Gamma_{j}, j=1, \ldots, 2(n+1)$, ordered in a clockwise sense, such that $A \nabla u \cdot \nu$ on $\Gamma_{j}$ is not allowed to be nonnegative for any even $j$ and is not allowed to be nonpositive for any odd $j, j=1, \ldots, 2(n+1)$.

Proof. We recall that $n$ is a nonnegative integer and it is positive if and only if $z_{0}$ is a geometrical critical point for $u$. We apply Proposition 2.1 to $z_{0}$. For any $j, j=1 \ldots, 2(n+1)$, let $\tilde{D}_{j}$ be the connected component of $\{z \in \Omega: u(z) \neq$ $0\}$ containing $D_{j}$. In either case, since clearly $k_{0}(\Sigma, \Omega, A, b) \leq k_{0}(\Omega \backslash \Sigma, A, b)$, we have that $k<k_{0}(\Omega \backslash \Sigma, A, b) \leq k_{0}\left(\tilde{D}_{j}, A, b\right)$ and so we can infer that $\left.u\right|_{\partial \tilde{D}_{j}}$ is not identically equal to zero. Obviously, $u=0$ for any point of $\partial \tilde{D}_{j} \cap \Omega$; therefore, there exists a point $y_{j} \in \partial \tilde{D}_{j} \cap \partial \Omega$ such that $u\left(y_{j}\right) \neq 0$. We can connect each $y_{j}$ with a point belonging to $D_{j}$ by a continuous curve which, but for its endpoint $y_{j}$, is contained in $\tilde{D}_{j}$. Therefore, since the sign of $u$ on $D_{j}$ (and hence also on $\tilde{D}_{j}$ ) changes from one $j$ to the next one, we can prove that the order of the points $y_{j}$ on $\partial \Omega$ is equal to the order defined by the sets $D_{j}$ and the sign of $u$ on $y_{j}$ is also changing from one $j$ to the next one. Here, and in the sequel, we clearly identify $j=2(n+1)+1$ with $j=1$. So, we have established part [ of the proposition.

For what concerns part [ii $)$, we consider the following reasoning. For any $j$, $j=1, \ldots, 2(n+1)$, we define the function $u_{j}$ in the following way:

$$
u_{j}= \begin{cases}u & \text { in } \tilde{D}_{j} \\ 0 & \text { in } \Omega \backslash \tilde{D}_{j} .\end{cases}
$$

We have that $u_{j} \in H^{1}(\Sigma, \Omega)$. Therefore, by the assumption on $k$, we obtain that $\int_{\Omega} A \nabla u_{j} \cdot \nabla u_{j}-k b u_{j}^{2}>0$. It is also easy to see that

$$
\int_{\Omega} A \nabla u_{j} \cdot \nabla u_{j}-k b u_{j}^{2}=\int_{\Omega} A \nabla u \cdot \nabla u_{j}-k b u u_{j}>0 .
$$

Therefore, we deduce that

$$
(A \nabla u \cdot \nu)\left[\left.u_{j}\right|_{\partial \Omega}\right]>0 \text { for any } j, j=1, \ldots, 2(n+1) .
$$


We have that $u_{j}$ is a continuous function on $\bar{\Omega}$ and is either nonpositive or nonnegative. By the previous construction, we are able to find $\Gamma_{j}, j=1, \ldots, 2(n+$ 1 ), open, connected and pairwise disjoint subarcs of $\partial \Omega$ such that $\left\{z \in \partial \Omega: u_{j}(z) \neq\right.$ $0\} \subset \Gamma_{j}$. As before, the arcs $\Gamma_{j}$ are ordered in a clockwise sense and preserve the order of the sets $D_{j}$ and $u$ changes sign from one $\Gamma_{j}$ to the adjacent ones. That is, we can assume, without loss of generality, that $u_{j}$ is nonnegative for $j$ even and nonpositive for $j$ odd, $j=1, \ldots, 2(n+1)$.

Let us assume that $u_{j}$ is nonnegative on $\Gamma_{j}$. If $A \nabla u \cdot \nu$ were also nonnegative on $\Gamma_{j}$, formula (2.31) would yield a contradiction. The case when $u_{j}$ is nonpositive on $\Gamma_{j}$ is treated in an analogous way; hence the proof is complete.

\section{The Main Uniqueness Results}

In this section we prove a unique determination of a multiple sound-soft defect buried into a domain $\Omega$ by a finite number of boundary measurements. We are interested in two cases. In the first one, Dirichlet conditions are assigned on the exterior boundary $\partial \Omega$ and the corresponding Neumann data are measured on an open subarc $\Gamma$ of $\partial \Omega$. In the second case, we prescribe Neumann conditions and we measure the corresponding Dirichlet data on $\Gamma$. A suitable choice of the data to be prescribed on $\partial \Omega$ allows us to uniquely determine the multiple defect.

Let us consider the following framework. Let $\Omega$ be a Lipschitz, bounded and simply connected domain. Let $A$ be a $2 \times 2$ measurable symmetric matrix defined in $\Omega$ and satisfying (2.11), and let $b$ be a measurable function in $\Omega$ satisfying (2.12), for a positive constant $\lambda$.

Let $\Sigma$ and $\Sigma^{\prime}$ be two multiple defects in $\Omega$. We fix also $\Gamma$, an open subarc of $\partial \Omega$.

We prove the following auxiliary proposition.

Proposition 3.1. Let $k$ satisfy $0<k<\min \left\{k_{0}(\Omega \backslash \Sigma, A, b), k_{0}\left(\Omega \backslash \Sigma^{\prime}, A, b\right)\right\}$.

Fix $\psi \in C(\bar{\Omega}) \cap H^{1}(\Omega)$ and let $u$ be the solution to (2.27) and let $u^{\prime}$ be the solution to the same problem when $\Sigma$ is replaced by $\Sigma^{\prime}$. Then if $A \nabla u \cdot \nu=A \nabla u^{\prime} \cdot \nu$ on $\Gamma$ in a weak sense, we have $u=u^{\prime}$ on $\Omega$.

For fixed $\eta \in L^{2}(\partial \Omega)$, if $v$ solves (2.28) and $v^{\prime}$ solves the same problem with $\Sigma$ replaced by $\Sigma^{\prime}$, and $v=v^{\prime}$ on $\Gamma$, then $v=v^{\prime}$ on $\Omega$.

Proof. We follow the arguments used to prove Proposition 3.1 in [2]. We define $G$ as the connected component of $\Omega \backslash\left(\Sigma \cup \Sigma^{\prime}\right)$ such that $\partial \Omega$ is contained in $\partial G$. By the unique continuation property that we have described in the previous section, we immediately obtain that $u=u^{\prime}$ in $G$ and indeed on $\bar{G}$, given the continuity of the functions involved. The same reasoning allows us to say that $v=v^{\prime}$ on $\bar{G}$. We need to check that $u=u^{\prime}$ and $v=v^{\prime}$ also outside $G$. We shall treat only the first case since the second is completely analogous.

On $\partial G \cap \Omega$ we have that $u=u^{\prime}=0$ since $\partial G \cap \Omega$ is clearly contained in $\Sigma \cup \Sigma^{\prime}$. Let $D$ be one of the connected components of $(\Omega \backslash \bar{G}) \backslash \Sigma$. The boundary of $D$ is composed of points either belonging to $\Sigma$ or to $\partial G \cap \Omega$. We infer that $u \equiv 0$ on $\partial D$ and this implies, given the assumption on $k$, that $u=0$ in $D$. Therefore, we have that $u=0$ on $\Omega \backslash \bar{G}$. By the same reasoning, we obtain that $u^{\prime}$ is equal to zero on $\Omega \backslash \bar{G}$ and therefore the two functions coincide everywhere.

Already this global unique continuation property provides us with a uniqueness result with a single measurement in some particular cases. In the first case, we consider a restriction on the kind of defects considered, namely, we assume that 
the unknown multiple defect coincides with the closure of its interior. With this assumption on $\Sigma$, one measurement will be enough to determine the multiple defect even if no assumption, but nontriviality, is imposed on the prescribed datum. In the second case, we do not restrict the kind of defects considered, but we impose the prescribed boundary datum to be nontrivial and either nonnegative or nonpositive on the whole $\partial \Omega$.

We describe these results in the following two theorems.

Theorem 3.2. Let $\Omega, A, b$ and $\Gamma$ satisfy the previously described assumptions.

Let $\Sigma$ and $\Sigma^{\prime}$ be two multiple defects in $\Omega$ such that $\Sigma=\stackrel{\bar{\circ}}{\Sigma}$, where $\stackrel{\circ}{\Sigma}$ denotes the interior part of $\Sigma$, and $\Sigma^{\prime}=\overline{\Sigma^{\prime}}$.

Let $k$ be such that $0<k<\min \left\{k_{0}(\Omega \backslash \Sigma, A, b), k_{0}\left(\Omega \backslash \Sigma^{\prime}, A, b\right)\right\}$. Let us fix $\psi \in$ $C(\bar{\Omega}) \cap H^{1}(\Omega)$ in such a way that $\left.\psi\right|_{\partial \Omega} \not \equiv 0$. Then if $u$, the solution to (2.27), and $u^{\prime}$, the solution to (2.27) with $\Sigma$ replaced by $\Sigma^{\prime}$, satisfy

$$
A \nabla u \cdot \nu=A \nabla u^{\prime} \cdot \nu \text { on } \Gamma,
$$

we have that $\Sigma=\Sigma^{\prime}$.

Let $k$ satisfy $0<k<\min \left\{k_{0}(\Sigma, \Omega, A, b), k_{0}\left(\Sigma^{\prime}, \Omega, A, b\right)\right\}$. For fixed $\eta \in L^{2}(\partial \Omega)$, $\eta \neq 0$, if $v$, the solution to (2.28), and $v^{\prime}$, the solution to (2.28) where $\Sigma$ is replaced by $\Sigma^{\prime}$, satisfy

then $\Sigma=\Sigma^{\prime}$.

$$
v=v^{\prime} \quad \text { on } \Gamma,
$$

Theorem 3.3. Let $\Omega, A, b$ and $\Gamma$ be as before. Let $\Sigma$ and $\Sigma^{\prime}$ be two multiple defects in $\Omega$.

We assume that $k$ satisfies $0<k<\min \left\{k_{0}(\Omega \backslash \Sigma, A, b), k_{0}\left(\Omega \backslash \Sigma^{\prime}, A, b\right)\right\}$. If $\psi \in$ $C(\bar{\Omega}) \cap H^{1}(\Omega)$ is such that $\left.\psi\right|_{\partial \Omega} \not \equiv 0$ and $\left.\psi\right|_{\partial \Omega} \geq 0$, then, letting $u$ and $u^{\prime}$ be defined as before, if

$$
A \nabla u \cdot \nu=A \nabla u^{\prime} \cdot \nu \quad \text { on } \Gamma,
$$

we have that $\Sigma=\Sigma^{\prime}$.

If $k$ is such that $0<k<\min \left\{k_{0}(\Sigma, \Omega, A, b), k_{0}\left(\Sigma^{\prime}, \Omega, A, b\right)\right\}$ and $\eta \in L^{2}(\partial \Omega)$, $\eta \neq 0$, is such that $\eta \geq 0$ on $\partial \Omega$, then, $v$ and $v^{\prime}$ being as in the previous theorem,

$$
v=v^{\prime} \text { on } \Gamma
$$

implies $\Sigma=\Sigma^{\prime}$.

Proof of Theorem 3.2 and Theorem 3.3. By Proposition 3.1 we infer, in both cases, that $u=u^{\prime}$ in $\Omega$ and $v=v^{\prime}$ in $\Omega$.

In order to prove Theorem 3.2 we argue by contradiction. Assume that $\Sigma \neq$ $\Sigma^{\prime}$. Then, without loss of generality, we can assume that $\Sigma^{\prime} \backslash \Sigma \neq \emptyset$ and, by our assumption on $\Sigma$ and $\Sigma^{\prime}, \Sigma^{\prime} \backslash \Sigma$ actually contains an open ball. On this ball, $u=$ $u^{\prime}=0$. Therefore, by unique continuation, $u=0$ in $\Omega$ and this would contradict the nontriviality of $\psi$. By the same reasoning, applied to $v$ and $v^{\prime}$, we obtain a proof for the second part of Theorem 3.2

Theorem 3.3 can be proved as follows. By Proposition 2.2 and the hypothesis on $\psi$ and $\eta$ respectively, we infer that $u$ and $v$ respectively are always different from zero inside $\Omega \backslash \Sigma$. Therefore, since $u=u^{\prime}$ in $\Omega$, or in the second case, since $v=v^{\prime}$ in $\Omega$, and $u^{\prime}=0$ on $\Sigma^{\prime}$, or $v^{\prime}=0$ on $\Sigma^{\prime}$ respectively, we deduce that $\Sigma^{\prime} \backslash \Sigma$ must be empty. Changing the roles of $\Sigma$ and $\Sigma^{\prime}$, we have that also $\Sigma \backslash \Sigma^{\prime}=\emptyset$ and hence $\Sigma=\Sigma^{\prime}$. 
Proposition 2.2 which describes the sign changes of the boundary data in terms of the index of a nodal geometrical critical point inside the medium, is crucial also for the proof of the other uniqueness result that we state in this paper. In this case, we make no assumptions on the multiple defect to be determined, but those given by the hypothesis on $k$. A single measurement, unless of the particular kind described in Theorem 3.3 would not be enough to uniquely determine the multiple defect, since we would not be able to decide whether a part of a nodal line of $u$ is contained in the multiple defect or not. Thus we need to take at least a second measurement. In the following theorem, we show that if we prescribe two suitably chosen boundary data, then the corresponding measurements uniquely identify the multiple defect.

Theorem 3.4. Let $\Omega, A, b$ and $\Gamma$ satisfy the previously described assumptions. Let $\Sigma$ and $\Sigma^{\prime}$ be two multiple defects in $\Omega$. Let $\gamma_{0}, \gamma_{1}$ and $\gamma_{2}$ be three open subarcs of $\partial \Omega$ that are pairwise disjoint.

Let $k$ be such that $0<k<\min \left\{k_{0}(\Omega \backslash \Sigma, A, b), k_{0}\left(\Omega \backslash \Sigma^{\prime}, A, b\right)\right\}$. Let $\psi_{1}$ and $\psi_{2}$ be two functions belonging to $C(\bar{\Omega}) \cap H^{1}(\Omega)$ and such that $\left.\psi_{1}\right|_{\partial \Omega}$ and $\left.\psi_{2}\right|_{\partial \Omega}$ are nontrivial, they coincide on $\gamma_{0}$, where both are nonincreasing, they are nondecreasing on $\gamma_{1}$ and $\gamma_{2}$ respectively, and are constant elsewhere.

Let $u_{1}$ and $u_{2}$ be the solutions to (2.27) with $\psi$ replaced by $\psi_{1}$ and $\psi_{2}$ respectively and let $u_{1}^{\prime}, u_{2}^{\prime}$ be the solutions to the same boundary value problems where $\Sigma$ is replaced by $\Sigma^{\prime}$. Then, if

$$
A \nabla u_{i} \cdot \nu=A \nabla u_{i}^{\prime} \cdot \nu \quad \text { on } \Gamma
$$

for any $i=1,2$, we have $\Sigma=\Sigma^{\prime}$.

Suppose that $k$ satisfies $0<k<\min \left\{k_{0}(\Sigma, \Omega, A, b), k_{0}\left(\Sigma^{\prime}, \Omega, A, b\right)\right\}$. Let $\eta_{1}$ and $\eta_{2}$ be two nontrivial $L^{2}(\partial \Omega)$ functions that coincide on $\gamma_{0}$, where both are nonnegative, are nonpositive on $\gamma_{1}$ and $\gamma_{2}$ respectively and are equal to zero elsewhere.

We set $v_{1}, v_{2}$ as the solutions to (2.28) with $\eta$ replaced by $\eta_{1}$ and $\eta_{2}$ respectively and $v_{1}^{\prime}, v_{2}^{\prime}$ as the solutions to the same boundary value problems if $\Sigma$ is replaced by $\Sigma^{\prime}$. Then, if

$$
v_{i}=v_{i}^{\prime} \quad \text { on } \Gamma
$$

for any $i=1,2$, we have $\Sigma=\Sigma^{\prime}$.

Proof. Let $\alpha, \beta$ be any two real numbers such that $\alpha^{2}+\beta^{2}=1$. Let $\psi=\alpha \psi_{1}+\beta \psi_{2}$. Then $u=\alpha u_{1}+\beta u_{2}$ solves (2.27), and we have that $u$ has no nodal geometrical critical points in $\Omega \backslash \Sigma$. In fact, we can find two open subarcs of $\partial \Omega$ such that $\psi$ is nonincreasing on the first one, nondecreasing on the second one and constant elsewhere. Therefore, for any value $c$ attained by $\psi$ on $\partial \Omega$, the set $\{z \in \partial \Omega$ : $\psi(z)=c\}$ has at most two connected components. Proposition 2.2 implies that if $u$ admits a nodal geometrical critical point, then the set $\{z \in \partial \Omega: u(z)=0\}$ has at least four connected components and this would contradict the configuration of $\psi$. By an analogous reasoning, we have that also $u^{\prime}=\alpha u_{1}^{\prime}+\beta u_{2}^{\prime}$ has no nodal geometrical critical points in $\Omega \backslash \Sigma^{\prime}$.

The second part of Proposition 2.2 provides, in an analogous way, that any linear combination of $v_{1}$ and $v_{2}$ (and of $v_{1}^{\prime}$ and $v_{2}^{\prime}$ respectively), with at least one coefficient different from zero, has no nodal geometrical critical points in $\Omega \backslash \Sigma\left(\Omega \backslash \Sigma^{\prime}\right.$ respectively).

Then the conclusion follows immediately from this claim, which we prove along the lines of the proof of Theorem 1.1 in [2]. 
Let $\omega_{1}$ and $\omega_{2}$ be two linearly independent functions satisfying (2.20) and, at the same time, the same equation (2.20) with $\Sigma$ replaced by $\Sigma^{\prime}$. Then, if $\Sigma^{\prime} \backslash \Sigma$ is not empty, we can find two real numbers $\alpha$, $\beta$, with $\alpha^{2}+\beta^{2}=1$, so that $\omega=\alpha \omega_{1}+\beta \omega_{2}$ has a nodal geometrical critical point in $\Omega \backslash \Sigma$.

Let $z_{0}$ belong to $\Sigma^{\prime} \backslash \Sigma$. Then $\omega_{i}\left(z_{0}\right)=0$ for any $i=1,2$. Let $r>0$ be such that $B_{r}\left(z_{0}\right) \subset \Omega \backslash \Sigma$ and let $w$ be the solution to (2.23) in $B_{r}\left(z_{0}\right)$. We consider the functions $\tilde{\omega}_{i}=\omega_{i} / w, i=1,2$, and their stream functions $\tilde{\theta}_{i}, i=1,2$. Without loss of generality, we can choose $\tilde{\theta}_{i}, i=1,2$, so that $\tilde{\theta}_{1}\left(z_{0}\right)=\tilde{\theta}_{2}\left(z_{0}\right)=0$. We take a sequence of points $\left\{z_{n}\right\}$ in $\Sigma^{\prime} \cap B_{r}\left(z_{0}\right)$, such that $z_{n}$ is different from $z_{0}$ for every $n$ and $z_{n}$ converges to $z_{0}$ as $n$ goes to infinity. We have that $\tilde{\omega}_{i}\left(z_{n}\right)=0$ for any $i=1,2$ and any $n$. For every $n$ we can choose $\alpha_{n}, \beta_{n}$ such that $\alpha_{n}^{2}+\beta_{n}^{2}=1$ and $\left(\alpha_{n} \tilde{\theta}_{1}+\beta_{n} \tilde{\theta}_{2}\right)\left(z_{n}\right)=0$. We can suppose, passing to a subsequence, that $\alpha_{n}$ and $\beta_{n}$ converge to $\alpha_{0}, \beta_{0}$ respectively as $n$ goes to infinity. We have that the function $\tilde{f}_{n}=\alpha_{n}\left(\tilde{\omega}_{1}+\mathrm{i} \tilde{\theta}_{1}\right)+\beta_{n}\left(\tilde{\omega}_{2}+\mathrm{i} \tilde{\theta}_{2}\right)$ has a zero in $z_{n}$ and in $z_{0}$. By the continuity property of the index, we obtain that $\tilde{f}_{0}=\alpha_{0}\left(\tilde{\omega}_{1}+\mathrm{i} \tilde{\theta}_{1}\right)+\beta_{0}\left(\tilde{\omega}_{2}+\mathrm{i} \tilde{\theta}_{2}\right)$ is such that $I\left(z_{0}, \tilde{f}_{0}\right) \geq 2$ and this implies that $z_{0}$ is a nodal geometrical critical point for $\omega=\alpha_{0} \omega_{1}+\beta_{0} \omega_{2}$.

We wish to make the following remarks about the assumptions on $k$ that we have used in Theorem 3.2. Theorem 3.3 and Theorem 3.4

The first assumption, that is, when we prescribe Dirichlet data, is that $k$ must satisfy $0<k<\min \left\{k_{0}(\Omega \backslash \Sigma, A, b), k_{0}\left(\Omega \backslash \Sigma^{\prime}, A, b\right)\right\}$. This hypothesis is satisfied for any multiple defect $\Sigma$ (including the empty set) if we assume $k<k_{0}(\Omega, A, b)$. Therefore, by (2.17), it will be enough to assume $0<k<\lambda^{2}(2 \pi /|\Omega|)^{1 / 2}$.

In the second assumption on $k$, when instead we prescribe Neumann data, we impose $0<k<\min \left\{k_{0}(\Sigma, \Omega, A, b), k_{0}\left(\Sigma^{\prime}, \Omega, A, b\right)\right\}$. First, we notice that this condition is stronger than the one considered above and also implies that $\Sigma$ and $\Sigma^{\prime}$ are not empty. Second, since the value of $k_{0}(\Sigma, \Omega, A, b)$, as we have seen in Section 2, can be bounded from below by a constant depending on $\lambda, \Omega$ and the Sobolev 2capacity of $\Sigma$ only (see (2.19)), any a priori information on the unknown multiple defect which allows us to estimate its capacity would provide also an estimate on the value of $k$ which can be used to apply our results. In particular, let us assume that the unknown multiple defect $\Sigma$ is such that $\delta(\Sigma)$ is greater than or equal to a fixed positive constant $\delta$. Then we have that $\Sigma$ is not empty and satisfies, using (2.10),

$$
C_{1,2}(\Sigma) \geq \frac{\pi}{40 \log (\max \{15,5 / \delta(\Sigma)\})} \geq \frac{\pi}{40 \log (\max \{15,5 / \delta\})}
$$

Hence there exists a positive constant $\tilde{k}$, depending on $\lambda, \Omega$ and $\delta$ only, such that $\tilde{k} \leq k_{0}(\Sigma, \Omega, A, b)$ for every $\Sigma$ such that $\delta(\Sigma) \geq \delta$. Therefore, if we further assume that $\Sigma$ and $\Sigma^{\prime}$ satisfy $\delta(\Sigma) \geq \delta$ and $\delta\left(\Sigma^{\prime}\right) \geq \delta$ respectively, $\delta$ being a fixed positive constant, then it will be enough to choose $k$ to be positive and less than the corresponding $\tilde{k}$.

We summarize these remarks in the following corollary.

Corollary 3.5. The conclusions of Theorem [3.2, Theorem 3.3 and Theorem 3.4 remain valid if we replace, in the Dirichlet case, the assumption that $k$ satisfies 


$$
\begin{aligned}
& 0<k<\min \left\{k_{0}(\Omega \backslash \Sigma, A, b), k_{0}\left(\Omega \backslash \Sigma^{\prime}, A, b\right)\right\} \text { with } \\
& \qquad 0<k<\lambda^{2}\left(\frac{2 \pi}{|\Omega|}\right)^{1 / 2} .
\end{aligned}
$$

In the Neumann case, if we further assume that $\delta(\Sigma) \geq \delta$ and $\delta\left(\Sigma^{\prime}\right) \geq \delta$, for a fixed positive constant $\delta$, then there exists a positive constant $C$ depending on $\Omega$ only such that if we replace the assumption that $k$ satisfies $0<k<$ $\min \left\{k_{0}(\Sigma, \Omega, A, b), k_{0}\left(\Sigma^{\prime}, \Omega, A, b\right)\right\}$ with

$$
0<k<\lambda^{2} C\left[\log \left(\max \left\{15, \frac{5}{\delta}\right\}\right)\right]^{-1 / 2},
$$

we have that the conclusions of Theorem 3.2 , Theorem 3.3 and Theorem 3.4 still hold.

\section{Applichtions to inverse Scattering}

In this section we describe how to obtain uniqueness results for the determination of sound-soft defects buried in an inhomogeneous and anisotropic medium by inverse scattering techniques, that is, by measuring far-field data. We shall show that the far-field data provide us information on the near field and, in turn, on boundary measurements. Applying the uniqueness results of the previous section, we are therefore able to uniquely determine a multiple sound-soft defect, of the most general type, in an inhomogeneous and anisotropic medium. Thus our results will be similar to the determination of obstacles inside an inhomogeneity described in [15, the main difference being the fact that we deal with the planar case and that we consider a much wider class of the (unknown) admissible multiple defects and of the (known) inhomogeneities surrounding them.

We begin by investigating the existence and uniqueness of the solution to our forward scattering problem. We shall essentially use the notation of [8], to which we refer also for a more comprehensive treatment of inverse scattering theory.

Let $A=A(z), z \in \mathbb{R}^{2}$, be a $2 \times 2$ symmetric matrix whose entries are measurable and $b=b(z), z \in \mathbb{R}^{2}$, be a measurable function. We assume that for a fixed positive constant $\lambda$ and for almost every $z \in \mathbb{R}^{2}, A$ satisfies the uniform ellipticity condition (2.11) and $b$ satisfies (2.12).

Roughly speaking, we assume that outside a bounded domain, the matrix $A$ coincides with the identity matrix and $b$ is identically equal to 1 . More precisely, we assume that there exists a positive constant $R$ such that

$$
\operatorname{supp}(A-I d) \subset B_{R}(0), \quad \operatorname{supp}(b-1) \subset B_{R}(0) .
$$

Let $k$ be a positive constant such that

$$
0<k<\frac{\sqrt{2} \lambda^{2}}{R}
$$

Finally, let $\Sigma$ be a multiple defect in $B_{R}(0)$, as defined in Section 2, page 220 .

Let the incident field be given by the time-harmonic acoustic plane wave

$$
u^{i}(z, t ; d)=\mathrm{e}^{\mathrm{i} \sqrt{k} z \cdot d-\omega t}
$$

where $\sqrt{k}$ is the wave number, $d$ is the direction of propagation and $\omega$ is the frequency. 
Then the direct scattering problem will be to find the total field $u(z ; d), z \in \mathbb{R}^{2}$, given by the sum of the incident field $u^{i}(z ; d)=\mathrm{e}^{\mathrm{i} \sqrt{k} z \cdot d}$ and of the scattered field $u^{s}(z ; d)$, which is due to the presence of the inhomogeneity and of the sound-soft multiple defect. The total field $u$ satisfies, in a weak sense, the problem

$$
\begin{cases}\operatorname{div}(A \nabla u)+k b u=0 & \text { in } \mathbb{R}^{2} \backslash \Sigma \\ u=0 & \text { on } \Sigma,\end{cases}
$$

and the so-called Sommerfeld radiation condition

$$
\lim _{r \rightarrow \infty} \sqrt{r}\left(\frac{\partial u^{s}}{\partial r}-\mathrm{i} \sqrt{k} u^{s}\right)=0 .
$$

By solving (4.2) in a weak sense, we mean that $u$ belongs to $C\left(\mathbb{R}^{2}\right) \cap H_{\text {loc }}^{1}\left(\mathbb{R}^{2}\right)$ and satisfies

$$
\int_{\mathbb{R}^{2} \backslash \Sigma} A \nabla u \cdot \nabla \phi-k b u \phi=0 \quad \text { for any } \phi \in C_{0}^{\infty}\left(\mathbb{R}^{2} \backslash \Sigma\right),
$$

and it is identically equal to zero on $\Sigma$. Concerning the Sommerfeld radiation condition, here $r=|z|$ and the limit has to be intended uniformly for all directions $z /|z|$.

From a physical point of view, the condition $u=0$ on $\Sigma$ models the presence of many sound-soft defects, whereas the Sommerfeld radiation condition characterizes outgoing waves and therefore characterizes the scattered wave.

We notice that outside $B_{R}(0)$, the Helmholtz type equation $\operatorname{div}(A \nabla u)+k b u=0$ coincides with the Helmholtz equation $\Delta u+k u=0$. Therefore, outside $B_{R}(0)$, the incident field $u^{i}$, the scattered field $u^{s}$ and the total field $u=u^{i}+u^{s}$ satisfy the Helmholtz equation $\Delta u+k u=0$.

The scattered field, since it satisfies the Sommerfeld condition, is said to be radiating and its asymptotic behaviour is one of an outgoing spherical wave. More precisely,

$$
u^{s}(z ; d)=\frac{\mathrm{e}^{\mathrm{i} \sqrt{k}|z|}}{\sqrt{|z|}}\left\{u_{\infty}^{s}(\hat{z} ; d)+O\left(\frac{1}{|z|}\right)\right\}
$$

as $|z|$ goes to $\infty$ uniformly in all directions $\hat{z}=z /|z|$.

The function $u_{\infty}^{s}(\cdot ; d)$, which is defined on the unit circle, is called the far-field pattern of $u^{s}$. The inverse scattering problem consists in identifying the multiple defect $\Sigma$ by measuring the far-field pattern of the scattered wave for one or more directions of propagation $d$ of the incident waves.

Existence and uniqueness of the solution to the direct problem (4.2)-(4.3) are an immediate consequence of the following proposition.

Proposition 4.1. Let $A, b, k$ and $\Sigma$ satisfy the previously stated assumptions for some constants $\lambda, R$. Let $g, f=\left(f_{1}, f_{2}\right)$ belong to $L^{p}\left(B_{R}(0)\right)$ and let $\psi$ belong to $C\left(B_{R}(0)\right) \cap W^{1, p}\left(B_{R}(0) \backslash \Sigma\right)$ for some $p>2$.

Then there exists a unique (weak) solution $u$ to the problem

$$
\begin{cases}\operatorname{div}(A \nabla u)+k b u=\operatorname{div}(f)+g & \text { in } \mathbb{R}^{2} \backslash \Sigma, \\ u=\psi & \text { on } \partial \Sigma, \\ \lim _{r \rightarrow \infty} \sqrt{r}\left(\frac{\partial u}{\partial r}-\mathrm{i} \sqrt{k} u\right)=0 . & \end{cases}
$$


Proof. By a weak solution to (4.5) we mean the following. We look for a function $u$ in $C\left(\mathbb{R}^{2}\right)$ such that $u \in H^{1}\left(B_{r}(0) \backslash \Sigma\right)$ for every $r>0$, and it satisfies

$$
\int_{\mathbb{R}^{2} \backslash \Sigma} A \nabla u \cdot \nabla \phi-k b u \phi=\int_{B_{R}(0)} f \cdot \nabla \phi-g \phi \quad \text { for any } \phi \in C_{0}^{\infty}\left(\mathbb{R}^{2} \backslash \Sigma\right),
$$

$u=\psi$, both in the classical and weak sense, on $\partial \Sigma$ and the Sommerfeld condition holds, where the limit has to be intended uniformly for all directions $z /|z|$ and $r=|z|$.

We can always assume, without loss of generality, that the support of $\psi$ is compactly contained in $B_{R}(0)$ and, possibly by choosing a slightly greater constant $R$ without breaking the assumption on $k$, that also $g$ and $f$ are compactly supported in $B_{R}(0)$. That is, we can suppose that there exists a constant $r, 0<r<R$, such that the following conditions hold. The multiple defect $\Sigma$ and the supports of $A-I d, b-1, \psi, g$ and $f$ are all (compactly) contained in $B_{r}(0)$. Let us denote by $h$ a cut-off function between $B_{r}(0)$ and $B_{R}(0)$, that is, a function belonging to $C_{0}^{\infty}\left(B_{R}(0)\right)$ that is identically equal to 1 in a neighbourhood of $\overline{B_{r}(0)}$.

The uniqueness of the solution relies on the following argument. By linearity, it will be enough to prove that if $\psi, g$ and $f$ are zero, then the function $u \equiv 0$ is the unique solution to the problem (4.5). So, let us assume, for the time being, that $u$ solves (4.5) with $\psi=0, g=0$ and $f=0$.

We have that

$$
\int_{B_{R}(0) \backslash \Sigma} A \nabla u \cdot \nabla \bar{u}-k b|u|^{2}=\int_{\partial B_{R}(0)} u \frac{\partial \bar{u}}{\partial \nu} .
$$

Hence the imaginary part of $\int_{\partial B_{R}(0)} u \frac{\partial \bar{u}}{\partial \nu}=0$ and this implies (see, for instance, 8]) that $u \equiv 0$ outside $B_{R}(0)$. Then, by unique continuation, $u$ is identically equal to zero in $\mathbb{R}^{2}$ and so uniqueness is established.

For what concerns existence we need to introduce the following notation. Let $\Phi(z, w)$ be the fundamental solution to the Helmholtz equation $\Delta u+k u=0$, which is defined as

$$
\Phi(z, w)=\frac{\mathrm{i}}{4} H_{0}^{(1)}(\sqrt{k}|z-w|), \quad z \neq w,
$$

where $H_{0}^{(1)}$ denotes the Hankel function of the first kind of order 0 ; see 8 for details. For any fixed $w \in \mathbb{R}^{2}, \Phi(\cdot, w)$ satisfies the Helmholtz equation in $\mathbb{R}^{2} \backslash\{w\}$. Given the fundamental solution, we define the acoustic single-layer potential as follows. For details and its basic properties we refer again to 8 .

Given a continuous function $\varphi$ on $\partial B_{R}(0)$, the function

$$
S \varphi(z)=\int_{\partial B_{R}(0)} \varphi(w) \Phi(z, w) \mathrm{d} s(w), \quad z \in \mathbb{R}^{2} \backslash \partial B_{R}(0),
$$

is called the single-layer potential with density $\varphi$.

We look for a solution constructed in the following way. We take a Hölder continuous function $\varphi$ on $\partial B_{R}(0)$, that is, $\varphi \in C^{\alpha}\left(\partial B_{R}(0)\right)$ for a given $\alpha, 0<\alpha<1$, and we define $v^{+}(z)=S \varphi(z)$ for every $z \in \mathbb{R}^{2} \backslash \overline{B_{R}(0)}$ and $v^{-}(z)=S \varphi(z)$ for every $z \in B_{R}(0)$.

We introduce the function $v$ as the (weak) solution to

$$
\begin{cases}\operatorname{div}(A \nabla v)+k b v=\operatorname{div}(f)+g & \text { in } B_{R}(0) \backslash \Sigma, \\ v=\psi & \text { on } \partial \Sigma, \\ v=v^{-} & \text {on } \partial B_{R}(0) .\end{cases}
$$


Then we look for a density $\varphi$ in such a way that the function $u$ defined as

$$
u(z)= \begin{cases}v^{+}(z) & \text { for every } z \in \mathbb{R}^{2} \backslash B_{R}(0), \\ v(z) & \text { for every } z \in B_{R}(0)\end{cases}
$$

solves the problem (4.5). The properties of the single-layer potential, its regularity and the regularity of the solution to the boundary value problem (4.6) imply that $u$ is a solution to (4.5) provided that the following transmission condition holds:

$$
\frac{\partial v^{+}}{\partial \nu}=\frac{\partial v}{\partial \nu} \quad \text { on } \partial B_{R}(0)
$$

We pick the auxiliary function $\tilde{v}=v-(1-h) v^{-}-\psi$, where $h$ is the cut-off function introduced before, and we rewrite the transmission condition (4.8) in the following form:

$$
\frac{\partial v^{+}}{\partial \nu}=\frac{\partial \tilde{v}}{\partial \nu}+\frac{\partial v^{-}}{\partial \nu}
$$

The behaviour of the single-layer potential on $\partial B_{R}(0)$ implies that, in order to solve problem (4.5), $\varphi$ must satisfy

$$
\varphi=-\frac{\partial \tilde{v}}{\partial \nu}
$$

We have that $\tilde{v}$ belongs to $H_{0}^{1}\left(B_{R}(0) \backslash \Sigma\right)$ and solves in $B_{R}(0) \backslash \Sigma$,

$$
\operatorname{div}(A \nabla \tilde{v})+k b \tilde{v}=\operatorname{div}(f)+g-(\Delta(1-h)) v^{-}-\operatorname{div}(A \nabla \psi)-k b \psi .
$$

Letting $\mathcal{L}$ be the elliptic operator defined on $H_{0}^{1}\left(B_{R}(0) \backslash \Sigma\right)$ such that $\mathcal{L}[u]=$ $\operatorname{div}(A \nabla u)+k b u$ in $B_{R}(0) \backslash \Sigma$, we have that this operator, by the assumption on $k$, is invertible. If we denote its inverse by $\mathcal{L}^{-1}$, then

$$
\tilde{v}=\mathcal{L}^{-1}\left[\operatorname{div}(f)+g-(\Delta(1-h)) v^{-}-\operatorname{div}(A \nabla \psi)-k b \psi\right] .
$$

So, we have that our construction provides a solution if $\varphi$ solves the equation

$$
\varphi+\frac{\partial}{\partial \nu}\left(\mathcal{L}^{-1}[(\Delta(h-1)) S \varphi]\right)=-\frac{\partial}{\partial \nu}\left(\mathcal{L}^{-1}[\operatorname{div}(f)+g-\operatorname{div}(A \nabla \psi)-k b \psi]\right) .
$$

In order to obtain an existence result for our problem (4.5), it is enough to show that (4.9) admits a solution. Standard regularity theory for elliptic equations implies that the right-hand side belongs to $C^{\alpha}\left(\partial B_{R}(0)\right)$ and the operator $K$ : $C^{\alpha}\left(\partial B_{R}(0)\right) \mapsto C^{\alpha}\left(\partial B_{R}(0)\right)$ defined by

$$
K \varphi=\frac{\partial}{\partial \nu}\left(\mathcal{L}^{-1}[(\Delta(h-1)) S \varphi]\right)
$$

is compact. Therefore, by the Fredholm theory for compact operators, it remains to prove that the equation $\varphi+K \varphi=0$ has a unique solution given by $\varphi=0$.

We observe that if $\varphi$ solves $\varphi+K \varphi=0$, then the function $u$, which is constructed as before, solves (4.5) with $\psi, g$ and $f$ equal to zero. The uniqueness previously obtained implies that $u$ would be identically equal to zero. Therefore, the singlelayer potential with density $\varphi$ will be identically equal to zero outside $B_{R}(0)$. From the continuity property of the single-layer potential, we deduce that $S \varphi$ is equal to zero on $\partial B_{R}(0)$ and satisfies the Helmholtz equation in $B_{R}(0)$. Our assumption on $k$ therefore implies that the single-layer potential with density $\varphi$ is zero everywhere in $\mathbb{R}^{2}$ and from this it is immediate to deduce that also $\varphi$ must be zero. 
Given existence and uniqueness of solutions to the direct problem (4.2)-(4.3), we can define the far-field operator $\mathcal{F}$ which associates to $d$, the direction of propagation of the incident field, the far-field pattern $u_{\infty}^{s}(\cdot ; d)$; that is,

$$
\mathcal{F}[d]=u_{\infty}^{s}(\cdot ; d) \quad \text { for every } d \text { in the unit circle. }
$$

It is clear that the far-field operator depends on the frequency (characterized by $k$ ), on the inhomogeneity (characterized by the coefficients of the Helmholtz type equation $A$ and $b$ ) and on the multiple defect $\Sigma$. Since we assume that the inhomogeneity and the frequency are given, whereas the defect is unknown, we shall state in an explicit way the dependence of the far-field operator from $\Sigma$, denoting it by $\mathcal{F}(\Sigma)$. Therefore, the inverse scattering problem that we shall investigate is of the following kind. We want to determine the shape and the location of $\Sigma$ by suitable information on the operator $\mathcal{F}(\Sigma)$.

First, we show that the far-field pattern determines the near field. Therefore, the measurement of the far-field pattern gives us corresponding boundary measurements on the boundary of $B_{R}(0)$. Before establishing this relation, let us introduce the operator that represents boundary measurements.

Given $A, b, k$ and $\Sigma$ satisfying the previously described assumptions, we know that for every $\psi \in H^{1 / 2}\left(\partial B_{R}(0)\right)$ the boundary value problem (2.27), with $\Omega$ replaced by $B_{R}(0)$, admits a unique solution $u$. We recall that the conormal derivative of $u$ on $\partial B_{R}(0),\left.A \nabla u \cdot \nu\right|_{\partial B_{R}(0)}$, is a well-defined element of $H^{-1 / 2}\left(\partial B_{R}(0)\right)$. The operator $\Lambda: H^{1 / 2}\left(\partial B_{R}(0)\right) \mapsto H^{-1 / 2}\left(\partial B_{R}(0)\right)$ such that

$$
\Lambda[\psi]=\left.A \nabla u \cdot \nu\right|_{\partial B_{R}(0)} \quad \text { for every } \psi \in H^{1 / 2}\left(\partial B_{R}(0)\right)
$$

where $u$ solves (2.27), will be called the Dirichlet-to-Neumann map. Again, this operator depends on $A, b, k, \Sigma$ and, obviously, $R$, but since we consider all the data except $\Sigma$ as fixed, we keep only this dependence and we denote the operator by $\Lambda(\Sigma)$.

Proposition 4.2. Let the previously stated assumptions on $A, b$ and $k$ be satisfied for some constants $\lambda$ and $R$ and let $\Sigma$ and $\Sigma^{\prime}$ be two multiple defects in $B_{R}(0)$. Fix a direction $d$, let $u$ be the solution to (4.2)-(4.3) and $u^{\prime}$ the solution to the same problem where $\Sigma$ is replaced by $\Sigma^{\prime}$. Then, if $\mathcal{F}(\Sigma)[d]=\mathcal{F}\left(\Sigma^{\prime}\right)[d]$, we have that $u=u^{\prime}$ on $\mathbb{R}^{2} \backslash B_{R}(0)$ and, if we set $\psi=\left.u\right|_{\partial B_{R}(0)}=\left.u^{\prime}\right|_{\partial B_{R}(0)}$, then also

$$
\Lambda(\Sigma)[\psi]=\Lambda\left(\Sigma^{\prime}\right)[\psi] .
$$

Proof. The scattered fields related to $\Sigma$ and $\Sigma^{\prime}$ respectively satisfy the same Helmholtz equation in $\mathbb{R}^{2} \backslash \overline{B_{R}(0)}$ and they also share the far-field pattern. Therefore, they coincide in $\mathbb{R}^{2} \backslash \overline{B_{R}(0)}$; see [ 8$]$. This, in turn, immediately implies that $u=u^{\prime}$ in $\mathbb{R}^{2} \backslash \overline{B_{R}(0)}$. Unique continuation allows us to prove that $u=u^{\prime}$ in an open neighbourhood of $\mathbb{R}^{2} \backslash B_{R}(0)$ and this concludes the proof of the proposition.

By using Proposition 3.1, it is immediate to notice that, under the assumptions of Proposition 4.2, $\mathcal{F}(\Sigma)[d]=\mathcal{F}\left(\Sigma^{\prime}\right)[d]$ implies also that $u=u^{\prime}$ everywhere in $\mathbb{R}^{2}$. Furthermore, we remark that $\psi=\left.u\right|_{\partial B_{R}(0)}=\left.u^{\prime}\right|_{\partial B_{R}(0)}$ is a nontrivial function. In fact, otherwise, the total field would be identically equal to zero, that is, $u^{s}=$ $-u^{i}$, and the Sommerfeld condition would be violated. Then as a corollary of Proposition 4.2, we obtain the following uniqueness result for the determination of a multiple obstacle. 
Theorem 4.3. Let $A, b$ and $k$ satisfy the previously described assumptions with constants $\lambda, R$.

Let $\Sigma$ and $\Sigma^{\prime}$ be two multiple defects in $B_{R}(0)$ such that $\Sigma=\stackrel{\bar{\circ}}{\Sigma}$ and $\Sigma^{\prime}=\overline{\Sigma^{\prime}}$. Then if $\mathcal{F}(\Sigma)[d]=\mathcal{F}\left(\Sigma^{\prime}\right)[d]$ for a fixed direction $d$, we have that $\Sigma=\Sigma^{\prime}$.

The relation between the far-field operator and the Dirichlet-to-Neumann map is indeed quite strong and we describe it in the following proposition.

Proposition 4.4. Given two positive constants $\lambda$ and $R$, let the previous assumptions on $A, b$ and $k$ be satisfied and let $\Sigma$ and $\Sigma^{\prime}$ be two multiple defects in $B_{R}(0)$.

Then we have that $\mathcal{F}(\Sigma)=\mathcal{F}\left(\Sigma^{\prime}\right)$ if and only if $\Lambda(\Sigma)=\Lambda\left(\Sigma^{\prime}\right)$.

Proof. We shall follow the lines of the proof of an analogous result contained in [14].

One of the two directions is easy. Namely, it is immediate to show that if the Dirichlet-to-Neumann maps are equal, so are the far-field operators. In fact, fix a direction $d$, let $u$ be the solution to the direct scattering problem (4.2)-(4.3) and let $\psi=\left.u\right|_{\partial B_{R}(0)}$. The fact that $\Lambda(\Sigma)=\Lambda\left(\Sigma^{\prime}\right)$, through unique continuation and the kind of reasoning used in the proof of Proposition [3.1, implies that $\left.u\right|_{B_{R}(0)}$, which is a solution in $B_{R}(0)$ of (2.27) with Dirichlet datum $\psi$, is also a solution of the same boundary value problem when $\Sigma$ is replaced by $\Sigma^{\prime}$. Therefore, we obtain, in turn, that $u$ solves also (4.2)- (4.3) when $\Sigma$ is replaced by $\Sigma^{\prime}$. By uniqueness of the solutions to the scattering problems, we have that the solutions to the two scattering problems, with $\Sigma$ and $\Sigma^{\prime}$ respectively, are equal, and therefore also their far-field patterns are the same.

The proof that $\mathcal{F}(\Sigma)=\mathcal{F}\left(\Sigma^{\prime}\right)$ implies $\Lambda(\Sigma)=\Lambda\left(\Sigma^{\prime}\right)$ relies on a density argument. For any direction of propagation $d,|d|=1$, of the incident field, let $u(\cdot ; d)$ be the solution to (4.2)-(4.3). It is clear from our previous results (in particular, from Proposition 4.2) that if $\psi=\left.u(\cdot ; d)\right|_{\partial B_{R}(0)}$, for a fixed direction $d$, then $\Lambda(\Sigma)[\psi]=$ $\Lambda\left(\Sigma^{\prime}\right)[\psi]$. Let $\operatorname{span}\left\{\left.u(\cdot ; d)\right|_{\partial B_{R}(0)}:|d|=1\right\}$ denote the subspace of $H^{1 / 2}\left(\partial B_{R}(0)\right)$ that is generated by the set $\left\{\left.u(\cdot ; d)\right|_{\partial B_{R}(0)}:|d|=1\right\}$. It is enough to prove that this subspace is dense in $H^{1 / 2}\left(\partial B_{R}(0)\right)$.

We fix a positive constant $\varepsilon_{0}$ such that $k$ satisfies also $0<k<\left(\sqrt{2} \lambda^{2}\right) /\left(R+\varepsilon_{0}\right)$ and $\Sigma$ and the supports of $A-I d$ and $b-1$ are contained in $B_{R-\varepsilon_{0}}(0)$.

Then we claim that for every $\varepsilon, 0<\varepsilon<\varepsilon_{0}$, if $u$ is a solution to

$$
\begin{cases}\operatorname{div}(A \nabla u)+k b u=0 & \text { in } B_{R+\varepsilon}(0) \backslash \Sigma \\ u=0 & \text { on } \Sigma\end{cases}
$$

then there exists a sequence of elements of $\operatorname{span}\{u(\cdot ; d):|d|=1\}$ converging to $u$ in $L^{2}\left(B_{R+\varepsilon / 2}(0) \backslash \overline{B_{R-\varepsilon / 2}(0)}\right)$.

First, we show that the claim immediately allows us to prove the density result needed. We take $\psi$ a real-analytic function on $\partial B_{R}(0)$, and we consider the solution $u$ to (2.27) with $\psi$ as boundary datum and $\Omega$ replaced by $B_{R}(0)$. By the analyticity of the boundary, the boundary datum and the coefficients of the Helmholtz type equation near the boundary of $B_{R}(0), u$ can be extended on a neighbourhood of $\overline{B_{R}(0)}$ in such a way that $u$ satisfies $\operatorname{div}(A \nabla u)+k b u=0$ in $B_{R+\varepsilon}(0) \backslash \Sigma$ for a positive $\varepsilon$. Therefore, in a neighbourhood of $\partial B_{R}(0)$, we can approximate $u$ in the $L^{2}$ norm by a sequence of elements of $\operatorname{span}\{u(\cdot ; d):|d|=1\}$. By standard regularity estimates in the interior for elliptic equations, we can find a, possibly smaller, neighbourhood of $\partial B_{R}(0)$ where $u$ can be approximated by a sequence of 
elements of $\operatorname{span}\{u(\cdot ; d):|d|=1\}$ in the $H^{1}$ norm. Therefore, every real-analytic function on $\partial B_{R}(0)$ can be approximated, in $H^{1 / 2}\left(\partial B_{R}(0)\right)$, by elements of the set $\operatorname{span}\left\{\left.u(\cdot ; d)\right|_{\partial B_{R}(0)}:|d|=1\right\}$ which, consequently, is dense in $H^{1 / 2}\left(\partial B_{R}(0)\right)$.

It remains to prove our claim, and we shall proceed as in the proof of Lemma 6.1.6 in 14. We argue by contradiction. If the claim is false, by the Hahn-Banach theorem, we can find a function $g \in L^{2}\left(B_{R+\varepsilon / 2}(0) \backslash \overline{B_{R-\varepsilon / 2}(0)}\right)$ such that $g$ is orthogonal to every element of $\operatorname{span}\{u(\cdot ; d):|d|=1\}$ but not to a solution $u$ to the problem (4.10). Let $v$ be the solution to (4.5) with $g$ as above, $f=0$ and $\psi=0$.

Then, if we set $r=R+\varepsilon$, we have that for every $d,|d|=1$,

$$
0=-\int_{B_{r}(0) \backslash \Sigma} g \bar{u}(\cdot ; d)=\int_{B_{r}(0) \backslash \Sigma}(A \nabla v \cdot \nabla \bar{u}(\cdot ; d)-k b v \bar{u}(\cdot ; d))-\int_{\partial B_{r}(0)} \frac{\partial v}{\partial \nu} \bar{u}(\cdot ; d) .
$$

Since also $u(\cdot ; d)$ solves the Helmholtz type equation in $B_{r}(0) \backslash \Sigma$, we obtain that

$$
\int_{\partial B_{r}(0)} \frac{\partial \bar{u}(\cdot ; d)}{\partial \nu} v-\frac{\partial v}{\partial \nu} \bar{u}(\cdot ; d)=0
$$

For any $\rho, \rho>r$, the functions $v, u^{i}(\cdot ; d)$ and $u^{s}(\cdot ; d)$ satisfy the Helmholtz equation in $B_{\rho}(0) \backslash \overline{B_{r}(0)}$, and $v$ and $u^{s}(\cdot ; d)$ satisfy also the Sommerfeld condition. Therefore, we have that

$$
\int_{\partial B_{r}(0)} \frac{\partial \overline{u^{s}}(\cdot ; d)}{\partial \nu} v-\frac{\partial v}{\partial \nu} \overline{u^{s}}(\cdot ; d)=\int_{\partial B_{\rho}(0)} \frac{\partial \overline{u^{s}}(\cdot ; d)}{\partial \nu} v-\frac{\partial v}{\partial \nu} \overline{u^{s}}(\cdot ; d)
$$

and, since by the Sommerfeld condition we deduce that

$$
\lim _{\rho \rightarrow \infty} \int_{\partial B_{\rho}(0)} \frac{\partial \overline{u^{s}}(\cdot ; d)}{\partial \nu} v-\frac{\partial v}{\partial \nu} \overline{u^{s}}(\cdot ; d)=0
$$

we obtain that

$$
\int_{\partial B_{r}(0)} \frac{\partial \overline{u^{i}}(\cdot ; d)}{\partial \nu} v-\frac{\partial v}{\partial \nu} \overline{u^{i}}(\cdot ; d)=0
$$

If we take $v_{0}$ as the solution in $B_{r}(0)$ of

$$
\begin{cases}\Delta v_{0}+k v_{0}=0 & \text { in } B_{r}(0), \\ v_{0}=v & \text { on } \partial B_{r}(0),\end{cases}
$$

we immediately have that, since $u^{i}(\cdot ; d)$ solves the same Helmholtz equation in $B_{R}(0)$,

and hence we deduce that

$$
\int_{\partial B_{r}(0)} \frac{\partial \overline{u^{i}}(\cdot ; d)}{\partial \nu} v_{0}-\frac{\partial v_{0}}{\partial \nu} \overline{u^{i}}(\cdot ; d)=0
$$

$$
\int_{\partial B_{r}(0)}\left(\frac{\partial v}{\partial \nu}-\frac{\partial v_{0}}{\partial \nu}\right) \bar{u}(\cdot ; d)=0
$$

Since the exponential solutions $\left\{u^{i}(\cdot ; d):|d|=1\right\}$ are dense in $L^{2}\left(\partial B_{r}(0)\right)$ (see [13]), we obtain that $v$ and $v_{0}$ share the same Cauchy data on $\partial B_{r}(0)$ and this implies that $v$ can be extended to a solution to the Helmholtz equation in the whole plane. By the fact that $v$ satisfies the radiation condition, we can deduce that $v$ is identically equal to zero outside $\overline{B_{R+\varepsilon / 2}(0)}$. With a reasoning similar to the one used before, we obtain that

$$
-\int_{B_{R+\varepsilon / 2}(0) \backslash \overline{B_{R-\varepsilon / 2}(0)}} g \bar{u}=-\int_{B_{r}(0) \backslash \Sigma} g \bar{u}=\int_{\partial B_{r}(0)} \frac{\partial \bar{u}}{\partial \nu} v-\frac{\partial v}{\partial \nu} \bar{u}=0,
$$


since $v$ is identically equal to zero in a neighbourhood of $\partial B_{r}(0)$. Therefore, $g$ should be orthogonal also to $u$ and this provides the contradiction required.

Proposition 4.4, with the aid of either Theorem 3.3 or of Theorem 3.4, implies immediately the following uniqueness result for the determination of a multiple defect by scattering data.

Theorem 4.5. For two positive fixed constants $\lambda$ and $R$, let the previously stated assumptions on $A, b$ and $k$ be satisfied.

Then if $\Sigma$ and $\Sigma^{\prime}$ are two multiple defects in $B_{R}(0)$ so that $\mathcal{F}(\Sigma)=\mathcal{F}\left(\Sigma^{\prime}\right)$, we have that $\Sigma=\Sigma^{\prime}$.

\section{REFERENCES}

[1] D. R. Adams and L. I. Hedberg, Function Spaces and Potential Theory, Springer-Verlag, Berlin, 1996. MR 97j:46024

[2] G. Alessandrini and A. Diaz Valenzuela, Unique determination of multiple cracks by two measurements, SIAM J. Control Optim. 34 (1996), pp. 913-921. MR 97a:78017

[3] G. Alessandrini and E. Di Benedetto, Determining 2-dimensional cracks in 3-dimensional bodies: uniqueness and stability, Indiana Univ. Math. J. 46 (1997), pp. 1-82. MR 98g:35207

[4] G. Alessandrini and R. Magnanini, Elliptic equations in divergence form, geometric critical points of solutions, and Stekloff eigenfunctions, SIAM J. Math. Anal. 25 (1994), pp. 12591268. MR 95f:35180

[5] L. Bers, F. John and M. Schechter, Partial Differential Equations, Interscience Publishers, New York, London, Sidney, 1964. MR 29:346

[6] L. Bers and L. Nirenberg, On a representation theorem for linear elliptic systems with discontinuous coefficients and its applications, in Convegno Internazionale sulle Equazioni Lineari alle Derivate Parziali, Trieste, Edizioni Cremonese, Roma, 1955, pp. 111-140. MR 17:974d

[7] D. Colton, J. Coyle and P. Monk, Recent developments in inverse acoustic scattering theory, SIAM Review 42 (2000), pp. 369-414. MR 2001f:76066

[8] D. Colton and R. Kress, Inverse Acoustic and Electromagnetic Scattering Theory, Second edition, Springer-Verlag, Berlin, 1998. MR 99c:35181

[9] A. Friedman and M. Vogelius, Determining cracks by boundary measurements, Indiana Univ. Math. J. 38 (1989), pp. 527-556. MR 91b:35109

[10] D. Gilbarg and N. S. Trudinger, Elliptic Partial Differential Equations of Second Order, Second edition, Springer-Verlag, Berlin, 1983. MR 86c:35035

[11] P. Hartman and A. Wintner, On the local behavior of solutions of non-parabolic partial differential equations, Amer. J. Math. 75 (1953), pp. 449-476. MR 15:318b

[12] J. Heinonen, T. Kilpeläinen and O. Martio, Nonlinear Potential Theory of Degenerate Elliptic Equations, Clarendon Press, Oxford Univ. Press, New York, 1993. MR 94e:31003

[13] V. Isakov, On uniqueness in the inverse transmission scattering problem, Comm. Partial Differential Equations 15 (1990), pp. 1565-1587. MR 91i:35203

[14] V. Isakov, Inverse Problems for Partial Differential Equations, Springer-Verlag, New York, 1998. MR 99b:35211

[15] A. Kirsch and L. Päivärinta, On recovering obstacles inside inhomogeneities, Math. Meth. Appl. Sci. 21 (1998), pp. 619-651. MR 99b:35214

[16] R. Kress, Inverse scattering from an open arc, Math. Meth. Appl. Sci. 18 (1995), pp. 267-293. MR 95k:35219

[17] J. R. Kuttler and V. G. Sigillito, Eigenvalues of the Laplacian in two dimensions, SIAM Review 26 (1984), pp. 163-193. MR 85k:65086

[18] W. Littman, G. Stampacchia and H. F. Weinberger, Regular points for elliptic equations with discontinuous coefficients, Ann. Scuola Norm. Sup. Pisa Cl. Sci. 17 (1963), pp. 43-77. MR 28: 4228

[19] N. G. Meyers, An $L^{p}$-estimate for the gradient of solutions of second order elliptic divergence equations, Ann. Scuola Norm. Sup. Pisa Cl. Sci. 17 (1963), pp. 189-206. MR 28:2328

[20] L. Rondi, Uniqueness and Optimal Stability for the Determination of Multiple Defects by Electrostatic Measurements, Ph.D. thesis, S.I.S.S.A.-I.S.A.S., Trieste, 1999 (downloadable from http://www.sissa.it/library/). 
[21] G. Stampacchia, Problemi al contorno ellittici, con dati discontinui, dotati di soluzioni hölderiane, Ann. Mat. Pura Appl. 51 (1960), pp. 1-37. MR 23:A3897

[22] M. Tsuji, Potential Theory in Modern Function Theory, Chelsea Publishing Company, New York, 1975, Reprint of the 1959 original. MR 54:2990

[23] W. P. Ziemer, Weakly Differentiable Functions, Springer-Verlag, New York, 1989. MR 91e:46046

School of Mathematics, University of Minnesota, Minneapolis, Minnesota 55455

Current address: Dipartimento di Scienze Matematiche, Università degli Studi di Trieste, Trieste, Italy

E-mail address: rondi@mathsun1.univ.trieste.it 